\title{
Hopf bifurcation for non-densely defined Cauchy problems
}

\author{
Zhihua Liu, Pierre Magal and Shigui Ruan
}

\begin{abstract}
In this paper, we establish a Hopf bifurcation theorem for abstract Cauchy problems in which the linear operator is not densely defined and is not a Hille-Yosida operator. The theorem is proved using the center manifold theory for nondensely defined Cauchy problems associated with the integrated semigroup theory. As applications, the main theorem is used to obtain a known Hopf bifurcation result for functional differential equations and a general Hopf bifurcation theorem for age-structured models.
\end{abstract}

Mathematics Subject Classification (2000). 34K18, 35K90, 37L10.

Keywords. Hopf bifurcation · Cauchy problem · Non-dense domain · Periodic solution · Functional differential equation · Age structured model.

\section{Introduction}

The Hopf bifurcation theorem, proved by several researchers (see Andronov et al. [3], Hopf [23], Friedrichs [16], Hale [18]), gives a set of sufficient conditions to ensure that an autonomous ordinary differential equation with a parameter exhibits non-trivial periodic solutions for certain values of the parameter. The theorem has been used to study bifurcations in many applied subjects (see Marsden and McCraken [38] and Hassard et al. [21]).

In the 1970s, several Hopf bifurcation theorems were obtained for infinite dimensional systems in order to establish the bifurcation of periodic solutions of the Navier-Stokes equations. Such results are usually based on the so-called Liapunov-Schmidt or center manifold reduction approach. We refer to Iudovich [26], Sattinger [41], Iooss [25], Joseph and Sattinger [27], Marsden [37], Marsden and McCraken [38], Crandall and Rabinowitz [9], Henry [22], Da Prato and Lunardi [12], and Kielhőfer [29] for results on the subject. The Hopf bifurcation theorem has also been extended to functional differential equations, see Hale [19], Hale and Verduyn Lunel [20], Diekmann et al. [14] and the references cited therein. We also refer to Golubitsky and Rabinowitz [17] for a nice commentary on Hopf bifurcation theorem and more references.

It has been observed that Hopf bifurcation also occurs in age-structured models (Prüss [40], Cushing [11], Swart [42], Kostova and Li [30], Bertoni [8]). Recently, we proved a Hopf bifurcation theorem for a specific class of age-structured model (Magal and Ruan [36]). However, there is still no general Hopf bifurcation theorem for general classes of age-structured models. This is one of our motivations for writing this paper.

Several types of differential equations, including functional differential equations, age-structured models in population dynamics and epidemiology, some partial differential equations, and evolution equations

Z. Liu, P. Magal-Research was partially supported by the French Ministry of Foreign and European Affairs program EGIDE (20932UL).

S. Ruan-Research was partially supported by the National Science Foundation (DMS-0715772 and DMS-1022728). 
with nonlinear boundary conditions, can be written as non-densely defined semilinear Cauchy problems (see Da Prato and Sinestrari [13], Thieme [43,44], Liu et al. [32], Magal and Ruan [34], and Chu et al. [10]). The main purpose of this paper is to present a general Hopf bifurcation theory for the non-densely defined abstract Cauchy problem:

$$
\frac{d u(t)}{d t}=A u(t)+F(\mu, u(t)), \quad \forall t \geq 0, \quad u(0)=x \in \overline{D(A)}
$$

where $A: D(A) \subset X \rightarrow X$ is a linear operator on a Banach space $X, F: \mathbb{R} \times \overline{D(A)} \rightarrow X$ is a $C^{k}$ map with $k \geq 2$, and $\mu \in \mathbb{R}$ is the bifurcation parameter. Here, we study the Cauchy problem (1.1) when $D(A)$ is not dense in $X$ and $A$ is not a Hille-Yosida operator. Also, the solutions must be understood as integrated solutions of (1.1), that is,

$$
\int_{0}^{t} u(s) d s \in D(A), \quad \forall t \geq 0
$$

and

$$
u(t)=x+A \int_{0}^{t} u(s) d s+\int_{0}^{t} F(\mu, u(s)) d s, \quad \forall t \geq 0 .
$$

We will apply the center manifold theorem developed in Magal and Ruan [36] to prove a Hopf bifurcation theorem for the abstract non-densely defined Cauchy problem (1.1). This result requires some clarification because it has not been proved elsewhere. Since the problem is written as a Cauchy problem, the method may seem fairly classical; however, the result is new and general, which can be applied to several types of equations as mentioned above. In fact, we will apply the main theorem to obtain a known Hopf bifurcation result for functional differential equations and a general Hopf bifurcation theorem for age-structured models. The latter is new and useful in studying the dynamics of age-structured models arisen in population dynamics and epidemiology.

The rest of the paper is organized as follows. In Sect. 2, we state the general Hopf bifurcation theorem for the non-densely defined Cauchy problem (1.1). In Sect. 3, the center manifold reduction theorem for non-densely defined Cauchy problems is recalled. In Sect. 4, the main result, Theorem 2.4, is proven. In Sect. 5, we apply Theorem 2.4 to establish Hopf bifurcation theorems for delay differential equations and age-structured models.

\section{Hopf bifurcation theorem}

Non-densely defined Cauchy problems were initiated by Da Prato and Sinestrari [13] using the idea of the sum of operator theory and by Arendt $[4,5]$ using the notion of integrated semigroups. In these earlier works on non-densely defined Cauchy problems, it was assumed that $A$ is a Hille-Yosida operator, that is, there exist two constants $\omega_{A} \in \mathbb{R}$ and $M_{A} \geq 1$ such that $\left(\omega_{A},+\infty\right) \subset \rho(A)$ and

$$
\left\|(\lambda I-A)^{-k}\right\|_{\mathcal{L}(X)} \leq \frac{M_{A}}{\left(\lambda-\omega_{A}\right)^{k}}, \forall \lambda>\omega_{A}, \quad \forall k \geq 1,
$$

where $\mathcal{L}(X)$ is the space of bounded linear operators from $X$ into $X$ and $\rho(A)$ is the resolvent set of $A$. 


\subsection{Assumptions}

To compare densely and non-densely defined Cauchy problems, we first consider the Cauchy problem

$$
\frac{d u(t)}{d t}=A u(t), \quad t \geq 0, \quad u(0)=x \in \overline{D(A)}
$$

The problem (2.1) is considered as a Cauchy problem on the closed subspace

$$
X_{0}:=\overline{D(A)} \text {. }
$$

Recall that $A_{0}: D\left(A_{0}\right) \subset X_{0} \rightarrow X_{0}$, the part of $A$ in $X_{0}$, is the linear operator on $X_{0}$ defined by

$$
\left.A_{0} x=A x \text { for each } x \in D\left(A_{0}\right)=\{x \in \overline{D(A)}): A x \in \overline{D(A)}\right\} .
$$

In order to define the solution of the Cauchy problem (2.1), we make the following assumption on the linear operator $A$.

Assumption 2.1. Assume that $A: D(A) \subset X \rightarrow X$ is a linear operator on a Banach space $(X,\|\|$.$) such$ that there exist two constants, $\omega_{A} \in \mathbb{R}$ and $M_{A} \geq 1$, such that $\left(\omega_{A},+\infty\right) \subset \rho(A)$ and the following properties are satisfied:

(a) $\lim _{\lambda \rightarrow+\infty}(\lambda I-A)^{-1} x=0, \forall x \in X$;

(b) $\left\|(\lambda I-A)^{-k}\right\|_{\mathcal{L}\left(X_{0}\right)} \leq \frac{M_{A}}{\left(\lambda-\omega_{A}\right)^{k}}, \forall \lambda>\omega_{A}, \quad \forall k \geq 1$.

One may observe that in Assumption 2.1 (b), the Hille-Yosida condition is satisfied only on the closure of the domain $X_{0}$. Assumption 2.1 implies in particular that $A_{0}$ is the infinitesimal generator of a strongly continuous semigroup $\left\{T_{A_{0}}(t)\right\}_{t \geq 0}$ of bounded linear operators on $X_{0}$ and

$$
\left\|T_{A_{0}}(t)\right\|_{\mathcal{L}\left(X_{0}\right)} \leq M_{A} e^{\omega_{A} t}, \quad \forall t \geq 0 .
$$

Moreover, since the resolvent set of $A$ is non-empty, we know (see for example Magal and Ruan [35, Lemma 2.1]) that

$$
\rho(A)=\rho\left(A_{0}\right)
$$

Next, we turn to the Cauchy problem

$$
\frac{d u(t)}{d t}=A u(t)+x, t \geq 0, \quad u(0)=0 .
$$

Using Assumption 2.1, we may explicitly define $\left\{S_{A}(t)\right\}_{t \geq 0}$, the integrated semigroup generated by $A$, as follows

$$
S_{A}(t)=\left(\mu I-A_{0}\right) \int_{0}^{t} T_{A_{0}}(s)(\mu I-A)^{-1} d s, \quad \forall t \geq 0, \forall \mu \in\left(\omega_{A},+\infty\right) .
$$

For each $t \geq 0, S_{A}(t)$ is a bounded linear operator on $X$ (since $(\mu I-A)^{-1}$ maps $X$ into $D(A)$ ). It turns out that for each $x \in X, t \rightarrow S_{A}(t) x$ is the unique integrated solution of the Cauchy problem (2.2). Then, we can consider the non-homogeneous Cauchy problem

$$
\frac{d u(t)}{d t}=A u(t)+f(t), \quad t \geq 0, \quad u(0)=x \in \overline{D(A)}
$$

for some $f \in L^{1}((0, \tau), X)$.

It is well known that the Cauchy problem (2.3) has at most one integrated solution (see Thieme [44]). Under Assumption 2.1 the existence becomes an issue. When $A$ is a Hille-Yosida operator, we have the 
following estimation

$$
\left\|S_{A}(t)-S_{A}(s)\right\| \leq M_{A} \int_{s}^{t} e^{\omega_{A} l} d l, \quad \forall t \geq s \geq 0 .
$$

In particular, the map $t \rightarrow S_{A}(t)$ is locally Lipschitz continuous in the norm of operators. Using this property, Kellermann and Hieber [28] proved that if $A$ is a Hille-Yosida operator, then for each $f \in$ $L^{1}((0, \tau), X)$ the map $t \rightarrow \int_{0}^{t} S_{A}(t-s) f(s) d s$ is continuously differentiable and

$$
u(t)=T_{A_{0}}(t) x+\frac{d}{d t} \int_{0}^{t} S_{A}(t-s) f(s) d s
$$

is the unique integrated solution of the Cauchy problem (2.3). We refer to Arendt [4,5], Neubrander [39], Kellermann and Hieber [28], Thieme [44], and Arendt et al. [6] for more detailed results on this topic.

Recently, the situation where $A$ is not a Hille-Yosida operator has been studied (see Magal and Ruan [34] and Thieme [47]). Here, we only assume that integrated solutions exist when $f \in C([0, \tau], X)$, so we make the following assumption.

Assumption 2.2. There exists a function $\delta:[0,+\infty) \rightarrow[0,+\infty)$ with

$$
\lim _{t(>0) \rightarrow 0} \delta(t)=0
$$

such that for each $\tau>0$ and $f \in C([0, \tau], X), t \rightarrow \int_{0}^{t} S_{A}(t-s) f(s) d s$ is continuously differentiable and

$$
\left\|\frac{d}{d t} \int_{0}^{t} S_{A}(t-s) f(s) d s\right\| \leq \delta(t) \sup _{s \in[0, t]}\|f(s)\|, \forall t \in[0, \tau] .
$$

One may observe that the property (2.4) in Assumption 2.2 plays a crucial role in obtaining some estimations for the solutions (see Magal and Ruan [35, Proposition 2.14]). Moreover, using the results of Thieme [47], Assumption 2.2 is equivalent to the fact that there exists $\tau>0$ such that

$$
V^{\infty}\left(S_{A}, 0, \tau\right)<+\infty, \quad \forall \tau>0, \quad \text { and } \quad \lim _{t(>0) \rightarrow 0} V^{\infty}\left(S_{A}, 0, t\right)=0
$$

where $V^{\infty}\left(S_{A}, 0, \tau\right)$ is the semivariation of $\left\{S_{A}(t)\right\}_{t \geq 0}$ on $[0, \tau]$ defined by

$$
V^{\infty}\left(S_{A}, 0, \tau\right):=\sup \left\{\left\|\sum_{i=1}^{n}\left(S_{A}\left(t_{i}\right)-S_{A}\left(t_{i-1}\right)\right) x_{i}\right\|\right\}<+\infty,
$$

in which the supremum is taken over all partitions $0=t_{0}<\ldots<t_{n}=\tau$ of the interval $[a, b]$ and over any $\left(x_{1}, \cdots, x_{n}\right) \in X^{n}$ with $\left\|x_{i}\right\|_{X} \leq 1, \forall i=1, \ldots, n$. We refer to Magal and Ruan [34] for some characterization of the existence of integrated solutions expressed in terms of the resolvent.

From here on, we set

$$
\left(S_{A} \diamond f\right)(t):=\frac{d}{d t} \int_{0}^{t} S_{A}(t-s) f(s) d s .
$$

Recently, a center manifold reduction theory has been developed by Magal and Ruan [36] for nondensely defined Cauchy problems satisfying Assumptions 2.1 and 2.2. Our goal in this article is to provide some general setting under which Hopf bifurcation occurs. In the sequel, we assume that 0 is an equilibrium for each $\mu \in \mathbb{R}$ small enough, that is,

$$
F(\mu, 0)=0, \quad \forall \mu \in \mathbb{R} .
$$


Moreover, replacing $A$ by $A+\partial_{x} F(0,0)$ and $F$ by $G(\mu, u(t))=F(\mu, x)-\partial_{x} F(0,0) x$, the problem is unchanged (since Theorem 3.1 in Magal and Ruan [34] implies that $A+\partial_{x} F(0,0)$ satisfies Assumptions 2.1 and 2.2). So without loss of generality, we can assume that

$$
\partial_{x} F(0,0)=0 .
$$

We make the following assumption.

Assumption 2.3. Let $\varepsilon>0$ and $F \in C^{k}\left((-\varepsilon, \varepsilon) \times B_{X_{0}}(0, \varepsilon) ; X\right)$ for some $k \geq 4$. Assume that the following conditions are satisfied:

(a) $F(\mu, 0)=0, \forall \mu \in(-\varepsilon, \varepsilon)$, and $\partial_{x} F(0,0)=0$.

(b) (Transversality condition) For each $\mu \in(-\varepsilon, \varepsilon)$, there exists a pair of conjugated simple eigenvalues of $\left(A+\partial_{x} F(\mu, 0)\right)_{0}$, denoted by $\lambda(\mu)$ and $\overline{\lambda(\mu)}$, such that

$$
\lambda(\mu)=\alpha(\mu)+i \omega(\mu),
$$

the map $\mu \rightarrow \lambda(\mu)$ is continuously differentiable,

$$
\omega(0)>0, \quad \alpha(0)=0, \quad \frac{d \alpha(0)}{d \mu} \neq 0,
$$

and

$$
\sigma\left(A_{0}\right) \cap i \mathbb{R}=\{\lambda(0), \overline{\lambda(0)}\} .
$$

(c) The essential growth rate of $\left\{T_{A_{0}}(t)\right\}_{t \geq 0}$ is strictly negative, that is,

$$
\omega_{0, \mathrm{ess}}\left(A_{0}\right)<0 \text {. }
$$

The above conditions are closely related to the usual conditions for the finite dimensional case. The only difference with respect to the finite dimensional case is assumption (c) that is necessary to deal with spectral theory of the semigroup generated by $A_{0}$.

\subsection{Hopf bifurcation theorem}

The main result of this article is the following theorem.

Theorem 2.4. (Hopf Bifurcation) Let Assumptions 2.1-2.3 be satisfied. Then, there exist a constant $\varepsilon^{*}>0$ and three $C^{k-1}$ maps, $\varepsilon \rightarrow \mu(\varepsilon)$ from $\left(0, \varepsilon^{*}\right)$ into $\mathbb{R}, \varepsilon \rightarrow x_{\varepsilon}$ from $\left(0, \varepsilon^{*}\right)$ into $\overline{D(A)}$, and $\varepsilon \rightarrow \gamma(\varepsilon)$ from $\left(0, \varepsilon^{*}\right)$ into $\mathbb{R}$, such that for each $\varepsilon \in\left(0, \varepsilon^{*}\right)$ there exists a $\gamma(\varepsilon)$-periodic function $u_{\varepsilon} \in C^{k}\left(\mathbb{R}, X_{0}\right)$, which is an integrated solution of (1.1) with the parameter value $\mu=\mu(\varepsilon)$ and the initial value $x=x_{\varepsilon}$. So for each $t \geq 0, u_{\varepsilon}$ satisfies

$$
u_{\varepsilon}(t)=x_{\varepsilon}+A \int_{0}^{t} u_{\varepsilon}(l) d l+\int_{0}^{t} F\left(\mu(\varepsilon), u_{\varepsilon}(l)\right) d l .
$$

Moreover, we have the following properties

(i) There exist a neighborhood $N$ of 0 in $X_{0}$ and an open interval $I$ in $\mathbb{R}$ containing 0 , such that for $\widehat{\mu} \in I$ and any periodic solution $\widehat{u}(t)$ in $N$ with minimal period $\widehat{\gamma}$ close to $\frac{2 \pi}{\omega(0)}$ of (1.1) for the parameter value $\widehat{\mu}$, there exists $\varepsilon \in\left(0, \varepsilon^{*}\right)$ such that $\widehat{u}(t)=u_{\varepsilon}(t+\theta)$ (for some $\left.\theta \in[0, \gamma(\varepsilon))\right), \mu(\varepsilon)=\widehat{\mu}$, and $\gamma(\varepsilon)=\widehat{\gamma}$.

(ii) The map $\varepsilon \rightarrow \mu(\varepsilon)$ is a $C^{k-1}$ function and we have the Taylor expansion 


$$
\mu(\varepsilon)=\sum_{n=1}^{\left[\frac{k-2}{2}\right]} \mu_{2 n} \varepsilon^{2 n}+O\left(\varepsilon^{k-1}\right), \quad \forall \varepsilon \in\left(0, \varepsilon^{*}\right),
$$

where $\left[\frac{k-2}{2}\right]$ is the integer part of $\frac{k-2}{2}$.

(iii) The period $\gamma(\varepsilon)$ of $t \rightarrow u_{\varepsilon}(t)$ is a $C^{k-1}$ function and

$$
\gamma(\varepsilon)=\frac{2 \pi}{\omega(0)}\left[1+\sum_{n=1}^{\left[\frac{k-2}{2}\right]} \gamma_{2 n} \varepsilon^{2 n}\right]+O\left(\varepsilon^{k-1}\right), \quad \forall \varepsilon \in\left(0, \varepsilon^{*}\right),
$$

where $\omega(0)$ is the imaginary part of $\lambda(0)$ defined in Assumption 2.3.

Remark 2.5. In Assumption 2.3, if we only assume that $k \geq 2$ and the condition (2.6) is replaced by

$$
\sigma\left(A_{0}\right) \cap i \omega(0) \mathbb{Z}=\{\lambda(0), \overline{\lambda(0)}\}
$$

(i.e. the spectrum of $A_{0}$ does not contain a multiple of $\lambda(0)$ ). Then, by the Hopf bifurcation theorem of Crandall and Rabinovitz [9], we deduce that the assertion (i) of Theorem 2.4 holds.

\section{Center manifold theorem}

The goal of this section is to recall and explain the center manifold theorem proved in Magal and Ruan [36]. We start by introducing some notations. Let $X$ and $Z$ be two Banach spaces. Denote by $\mathcal{L}(X, Z)$ the space of bounded linear operators from $X$ into $Z$ and by $\mathcal{L}(X)$ the space $\mathcal{L}(X, X)$. Let $A: D(A) \subset X \rightarrow X$ be a linear operator. We denote by $\mathcal{R}(A)$ the range of $A, \mathcal{N}(A)$ the null space of $A$. If $A$ is the infinitesimal generator of a strongly continuous semigroup of bounded linear operators on $X$ (respectively, the generator of an integrated semigroup), we denote by $\left\{T_{A}(t)\right\}_{t \geq 0}$ this semigroup (respectively, $\left\{S_{A}(t)\right\}_{t>0}$ this integrated semigroup). Recall that $A$ is invertible if $A$ is a bijection from $D(A)$ into $X$ and $A^{-1}$ is bounded. If $X$ is a $\mathbb{C}$-Banach space, we recall that the resolvent set of $A$ is defined by $\rho(A)=\{\lambda \in \mathbb{C}: \lambda I-A$ is invertible $\}$. Moreover, we denote by $\sigma(A):=\mathbb{C} \backslash \rho(A)$ the spectrum of $A$. Let $Y$ be a subspace of $X$. $Y$ is said to be invariant by $A$ if

$$
A(D(A) \cap Y) \subset Y .
$$

Denote by $\left.A\right|_{Y}: D\left(\left.A\right|_{Y}\right) \subset Y \rightarrow X$ the restriction of $A$ to $Y$, which is defined by

$$
\left.A\right|_{Y} x=A x, \quad \forall x \in D\left(\left.A\right|_{Y}\right)=D(A) \cap Y .
$$

Denote by $A_{Y}: D\left(A_{Y}\right) \subset Y \rightarrow Y$ the part of $A$ in $Y$, which is defined by

$$
A_{Y} x=A x, \quad \forall x \in D\left(A_{Y}\right)=\{x \in D(A) \cap Y: A x \in Y\} .
$$

As before, we define

$$
X_{0}:=\overline{D(A)} \text { and } A_{0}:=A_{X_{0}} .
$$

For a given bounded linear operator $L \in \mathcal{L}(X),\|L\|_{\text {ess }}$ is the essential norm of $L$ defined by

$$
\|L\|_{\mathrm{ess}}=\kappa\left(L\left(B_{X}(0,1)\right)\right),
$$

here $B_{X}(0,1)=\left\{x \in X:\|x\|_{X} \leq 1\right\}$, and for each bounded set $B \subset X, \kappa(B)=\inf \{\varepsilon>0: B$ can be covered by a finite number of balls of radius $\leq \varepsilon\}$ is the Kuratovsky measure of non-compactness. 
Definition 3.1. Let $L: D(L) \subset X \rightarrow X$ be the infinitesimal generator of a linear $C_{0}$-semigroup $\left\{T_{L}(t)\right\}_{t \geq 0}$ on a Banach space $X$. Define the growth bound $\omega_{0}(L) \in[-\infty,+\infty)$ of $L$ by

$$
\omega_{0}(L):=\lim _{t \rightarrow+\infty} \frac{\ln \left(\left\|T_{L}(t)\right\|_{\mathcal{L}(X)}\right)}{t} .
$$

The essential growth bound $\omega_{0, \text { ess }}(L) \in[-\infty,+\infty)$ of $L$ is defined by

$$
\omega_{0, \mathrm{ess}}(L):=\lim _{t \rightarrow+\infty} \frac{\ln \left(\left\|T_{L}(t)\right\|_{\mathrm{ess}}\right)}{t} .
$$

Assumption 3.2. Assume that Assumptions 2.1-2.2 are satisfied and there exist two bounded linear operators of projection with finite rank, $\Pi_{0 c} \in \mathcal{L}\left(X_{0}\right) \backslash\{0\}$ and $\Pi_{0 u} \in \mathcal{L}\left(X_{0}\right)$, such that

$$
\Pi_{0 c} \Pi_{0 u}=\Pi_{0 u} \Pi_{0 c}=0
$$

and

$$
\Pi_{0 k} T_{A_{0}}(t)=T_{A_{0}}(t) \Pi_{0 k}, \quad \forall t \geq 0, \quad \forall k=\{c, u\} .
$$

Assume in addition that

(a) If $\Pi_{0 u} \neq 0$, then $\omega_{0}\left(-\left.A_{0}\right|_{\Pi_{0 u}\left(X_{0}\right)}\right)<0$.

(b) $\sigma\left(\left.A_{0}\right|_{\Pi_{0 c}\left(X_{0}\right)}\right) \subset i \mathbb{R}$.

(c) If $\Pi_{0 s}:=I-\left(\Pi_{0 c}+\Pi_{0 u}\right) \neq 0$, then $\omega_{0}\left(\left.A_{0}\right|_{\Pi_{0 s}\left(X_{0}\right)}\right)<0$.

In Assumption 3.2 we assumed that there is a state space decomposition of $X_{0}$

$$
X_{0}=X_{0 s} \oplus X_{0 c} \oplus X_{0 u},
$$

which corresponds, respectively, to the stable $X_{0 s}=\Pi_{0 s}\left(X_{0}\right)$, center $X_{0 c}=\Pi_{0 c}\left(X_{0}\right)$, and unstable $X_{0 u}=\Pi_{0 u}\left(X_{0}\right)$ linear manifold for the linear operator $A_{0}$. We assume that $X_{0 c}$ and $X_{0 u}$ have a finite dimension and are positively invariant by the resolvent of $A_{0}$. Moreover, we assume that

$$
\sigma\left(\left.A_{0}\right|_{\Pi_{0 u}\left(X_{0}\right)}\right) \subset\{\lambda \in \mathbb{C}: \operatorname{Re}(\lambda)>0\}
$$

and

$$
\sigma\left(\left.A_{0}\right|_{\Pi_{0 c}\left(X_{0}\right)}\right) \subset i \mathbb{R} .
$$

Furthermore, we assume that the growth rate of $\left\{\left.T_{A_{0}}(t)\right|_{\Pi_{0 s}\left(X_{0}\right)}\right\}_{t \geq 0}$ (which is the linear semigroup on $\Pi_{0 s}\left(X_{0}\right)$ generated by $A_{0 \Pi_{0 s}\left(X_{0}\right)}$ the part of $A_{0}$ in $\left.\Pi_{0 s}\left(X_{0}\right)\right)$ is negative. One may observe that under Assumptions 2.1-2.2, Assumption 3.2 is satisfied if and only if the following two properties hold:

(a) The essential growth rate of $\omega_{0, \text { ess }}\left(A_{0}\right)<0$;

(b) $\sigma\left(A_{0}\right) \cap i \mathbb{R} \neq \emptyset$.

Since the ranks of $\Pi_{0 c}$ and $\Pi_{0 u}$ are finite, we can apply Proposition 3.5 in Magal and Ruan [36] and deduce that for each $k=\{c, u\}$, there exists a unique bounded linear operator $\Pi_{k}: X \rightarrow X$ on $X$ satisfying:

(i) $\left.\Pi_{k}\right|_{X_{0}}=\Pi_{0 k}$;

(ii) $\Pi_{k}(X) \subset X_{0}$;

(iii) $\Pi_{k}(\lambda I-A)^{-1}=(\lambda I-A)^{-1} \Pi_{k}, \forall \lambda>\omega_{A}$.

Set

$$
\Pi_{s}=I-\left(\Pi_{c}+\Pi_{u}\right) \text { and } \Pi_{h}=I-\Pi_{c} .
$$

Then, we have for each $k \in\{c, h, s, u\}$ that

$$
\begin{aligned}
& \Pi_{k}(\lambda I-A)^{-1}=(\lambda I-A)^{-1} \Pi_{k}, \quad \forall \lambda>\omega_{A}, \\
& \Pi_{k}\left(X_{0}\right) \subset X_{0},
\end{aligned}
$$


and for each $k \in\{c, u\}$ that

$$
\Pi_{k}(X) \subset X_{0} .
$$

For each $k \in\{c, h, s, u\}$, set

$$
X_{0 k}=\Pi_{0 k}\left(X_{0}\right), \quad X_{k}=\Pi_{k}(X), \quad A_{k}=\left.A\right|_{X_{k}}=A_{X_{k}}, \text { and } A_{0 k}=\left.A_{0}\right|_{X_{0 k}}=A_{X_{0 k}} .
$$

Then, for each $k \in\{c, u\}$, we have

$$
X_{k}=X_{0 k} .
$$

The point with the above construction is that if $t \rightarrow u(t)$ is a solution of

$$
u(t)=x+A \int_{0}^{t} u(s) d s+\int_{0}^{t} f(s) d s, \quad t \geq 0,
$$

for some $x \in \overline{D(A)}$ and some $f \in C([0,+\infty), X)$, using the results obtained in Magal and Ruan [36, Chapter 3], we know that for each $k \in\{c, h, s, u\}$, the linear operator $A_{k}: D\left(A_{k}\right) \subset X_{k} \rightarrow X_{k}$ (with $\left.D\left(A_{k}\right)=X_{k} \cap D(A)\right)$ satisfies Assumptions 2.1-2.2, and $t \rightarrow \Pi_{k} u(t)$ is an integrated solution of the Cauchy problem

$$
\frac{d \Pi_{k} u(t)}{d t}=A_{k} \Pi_{k} u(t)+\Pi_{k} f(t), \quad t \geq 0, \quad \Pi_{k} u(0)=\Pi_{k} x,
$$

that is, $t \rightarrow \Pi_{k} u(t)$ satisfies

$$
\Pi_{k} u(t)=\Pi_{k} x+A_{k} \int_{0}^{t} \Pi_{k} u(s) d s+\int_{0}^{t} \Pi_{k} f(s) d s .
$$

In particular, if $k \in\{c, u\}$, since $X_{0 k}=X_{k}$, we have $A_{k}=A_{0 k}$. Moreover, since $\operatorname{dim}\left(X_{0 k}\right)<+\infty$, the linear operator $A_{0 k}$ is a bounded linear operator, so $t \rightarrow \Pi_{k} u(t)$ satisfies the following ordinary differential equation

$$
\frac{d \Pi_{k} u(t)}{d t}=A_{0 k} \Pi_{k} u(t)+\Pi_{k} f(t), \quad \forall t \geq 0, \quad \Pi_{k} u(0)=\Pi_{k} x .
$$

We define $C_{b}^{k}\left(X_{0 c}, X_{0 h}\right)$ as the set of bounded maps from $X_{0 c}$ into $X_{0 h}$ which are $k$-time continuously differentiable and all derivatives are bounded maps. The following result is proved in Magal and Ruan [36, Theorem 4.21]. This theorem is inspired by Theorem 3 in Vanderbauwhede and Iooss [48].

Theorem 3.3. (Local Center Manifold) Let Assumption 3.2 be satisfied. Let $r>0$ and $F: B_{X_{0}}(0, r) \rightarrow X$ be a map. Assume that there exists an integer $k \geq 1$ such that $F$ is $k$-time continuously differentiable in $B_{X_{0}}(0, r)$ with $F(0)=0$ and $D F(0)=0$. Then, there exist a neighborhood $\Omega$ of the origin in $X_{0}$ and a map $\Psi \in C_{b}^{k}\left(X_{0 c}, X_{0 h}\right)$, with $\Psi(0)=0$ and $D \Psi(0)=0$, such that the following properties hold:

(i) If $I$ is an interval of $\mathbb{R}$ and $x_{c}: I \rightarrow X_{0 c}$ is a solution of

$$
\frac{d x_{c}(t)}{d t}=A_{0 c} x_{c}(t)+\Pi_{c} F\left(x_{c}(t)+\Psi\left(x_{c}(t)\right)\right)(\text { reduced equation })
$$

such that

$$
u(t):=x_{c}(t)+\Psi\left(x_{c}(t)\right) \in \Omega, \quad \forall t \in I,
$$

then for each $t, s \in I$ with $t \geq s$,

$$
u(t)=u(s)+A \int_{s}^{t} u(l) d l+\int_{s}^{t} F(u(l)) d l .
$$


(ii) If $u: \mathbb{R} \rightarrow X_{0}$ is a map such that for each $t, s \in \mathbb{R}$ with $t \geq s$,

$$
u(t)=u(s)+A \int_{s}^{t} u(l) d l+\int_{s}^{t} F(u(l)) d l
$$

and

$$
u(t) \in \Omega, \quad \forall t \in \mathbb{R},
$$

then

$$
\Pi_{h} u(t)=\Psi\left(\Pi_{c} u(t)\right), \quad \forall t \in \mathbb{R},
$$

and $\Pi_{c} u: \mathbb{R} \rightarrow X_{0 c}$ is a solution of (3.1).

A first key tool to prove the above theorem is the truncation method used by Vanderbauwhede and Iooss [48]. One may observe that in order to apply such a truncation method, we need to assume that the dimension of $X_{0 c} \oplus X_{0 u}$ is finite. One may also observe that in order to define the reduced Eq. (3.1) we need to extend the projector $\Pi_{0 c}: X_{0} \rightarrow X_{0}$, because $F$ maps $X_{0}:=\overline{D(A)}$ into $X$ in general. So Proposition 3.5 in Magal and Ruan [36] also plays an important role to define the reduced Eq. (3.1).

In order to apply Theorem 3.3, we only need to define the semiflow generated by the Cauchy problem

$$
\frac{d u(t)}{d t}=A u(t)+F(u(t)), \quad t \geq 0, \quad u(0)=x \in \overline{D(A)}
$$

locally around 0 . We assume that $F$ is Lipschiz continuous. Consider a truncation function $\rho \in$ $C^{\infty}([0,+\infty),[0,+\infty))$ defined by

$$
\rho(x)= \begin{cases}0, & \text { if } x \geq 2 \\ \in[0,1], & \text { if } x \in[1,2] \\ 1, & \text { if } x \in[0,1]\end{cases}
$$

and define $F_{r}: \overline{D(A)} \rightarrow X$ by

$$
F_{r}(X)= \begin{cases}\rho\left(r^{-1}\|x\|\right) F(x), & \text { if }\|x\| \leq \varepsilon \\ 0, & \text { if }\|x\| \geq \varepsilon .\end{cases}
$$

Then, we have the following properties:

(i) $F_{r}(X)=F(x)$ for each $x \in B_{X_{0}}(0, r)$ and each $r \in(0, \varepsilon]$;

(ii) There exists $r_{0}>0$ such that $F_{r} \in \operatorname{Lip}\left(X_{0}, X\right)$ for each $r \in\left(0, r_{0}\right]$;

(iii) $\lim _{r(>0) \rightarrow 0}\left\|F_{r}\right\|_{\text {Lip }}=0$.

Let $r^{*} \in\left(0, r_{0}\right]$ such that

$$
B_{X_{0}}\left(0, r^{*}\right) \subset \Omega .
$$

Since $F_{r^{*}}$ is Lipschitz continuous, we can define a unique nonlinear semiflow $\{U(t)\}_{t \geq 0}$ on $X_{0}$, such that for each $x \in X_{0}, t \rightarrow U(t) x$ is the unique integrated solution of the Cauchy problem

$$
\frac{d U(t) x}{d t}=A U(t) x+F_{r^{*}}(U(t) x), \quad t \geq 0, \quad U(0) x=x \in X_{0},
$$

or equivalently $t \rightarrow U(t) x$ is the unique continuous map from $[0,+\infty)$ into $X_{0}$ satisfying the fixed point problem

$$
U(t) x=T_{A_{0}}(t) x+\left(S_{A} \diamond F_{r^{*}}(U(.) x)\right)(t), \quad \forall t \geq 0 .
$$

Now, since $F$ and $F_{r^{*}}$ coincide on $B_{X_{0}}\left(0, r^{*}\right)$, we deduce by applying Theorem 3.3-(ii) that if $\{u(t)\}_{t \in \mathbb{R}} \subset B_{X_{0}}\left(0, r^{*}\right)$ is a complete orbit of $\{U(t)\}_{t \geq 0}$, that is,

$$
u(t)=U(t-s) u(s), \quad \forall t, s \in \mathbb{R} \text { with } t \geq s,
$$


then

$$
u(t) \in M, \forall t \in \mathbb{R},
$$

where the center manifold $M \subset X_{0}$ is defined as

$$
M:=\left\{x_{c}+\Psi\left(x_{c}\right): x_{c} \in X_{0 c}\right\} \subset X_{0} .
$$

Now, by projecting $u(t)$ on $X_{0 c}$ we deduce that $t \rightarrow \Pi_{c} u(t)$ satisfies the reduced equation

$$
\frac{d \Pi_{c} u(t)}{d t}=A_{0 c} \Pi_{c} u(t)+\Pi_{c} F\left(\Pi_{c} u(t)+\Psi\left(\Pi_{c} u(t)\right)\right) .
$$

In particular, if $u(t)=\bar{x}$ is an equilibrium of (3.3) in $B_{X_{0}}\left(0, r^{*}\right)$, or if $t \rightarrow u(t)$ is a periodic solution of (3.3) in $B_{X_{0}}\left(0, r^{*}\right)$, then $t \rightarrow \Pi_{c} u(t)$ is a solution of the reduced Eq. (3.1).

Conversely, since the first derivative of $\Psi$ is bounded, we deduce that $\Psi$ is Lipschitz continuous, and since $\Psi(0)=0$, we can find $r_{1} \in\left(0, r^{*}\right)$ such that for each $x_{c} \in B_{X_{c}}\left(0, r_{1}\right)$, we have

$$
\left\|x_{c}+\Psi\left(x_{c}\right)\right\| \leq r^{*} .
$$

Now if $t \rightarrow x_{c}(t)$ is a global solution of the reduced Eq. (3.1) such that

$$
\left\|x_{c}(t)\right\| \leq r_{1}, \quad \forall t \in \mathbb{R},
$$

by applying Theorem 3.3-(i), we deduce that $t \rightarrow x_{c}(t)+\Psi\left(x_{c}(t)\right)$ is a complete orbit of $\{U(t)\}_{t \geq 0}$ in $B_{X_{0}}\left(0, r^{*}\right)$. In particular, $t \rightarrow u(t)$ is an integrated solution of the abstract Cauchy problem (3.2).

In order to prove the Hopf bifurcation theorem, we need an additional result about the spectral properties of the linearized equation around an equilibrium. Assume that $\bar{x} \in B_{X_{0}}\left(0, r^{*}\right)$ is an equilibrium of (3.2) (i.e., $\bar{x} \in D(A)$ and $A \bar{x}+F(\bar{x})=0$ ). Then (see Magal and Ruan [35]), since $F$ is differentiable around $\bar{x}$, we know that $x \rightarrow U(t) x$ is differentiable. Moreover, $V(t)=\partial_{x} U(t)(\bar{x})$ is a solution of the Cauchy problem

$$
\frac{d v(t)}{d t}=A v(t)+D F(\bar{x})(v(t)), t \geq 0, \quad v(0)=y \in \overline{D(A)} .
$$

More precisely, $\{V(t)\}_{t \geq 0}$ is the strongly continuous semigroup of bounded linear operators on $\overline{D(A)}$ generated by $(A+D F(\bar{x}))_{0}$, the part of $A+D F(\bar{x}): D(A) \subset X \rightarrow X$ in $\overline{D(A)}$. Now, since $\bar{x} \in$ $B_{X_{0}}\left(0, r^{*}\right), \bar{x}_{c}=\Pi_{0 c} \bar{x}$ is also an equilibrium of the reduced Eq. (3.1). Moreover, the linearized equation of (3.1) around $\bar{x}_{c}$ is given by

$$
\frac{d y_{c}(t)}{d t}=A_{0 c} y_{c}(t)+\Pi_{c} D F\left(\bar{x}_{c}+\Psi\left(\bar{x}_{c}\right)\right)\left(y_{c}(t)+D \Psi\left(\bar{x}_{c}\right)\left(y_{c}(t)\right)\right):=L\left(y_{c}(t)\right) .
$$

The goal of the following proposition is to describe the relationship between the spectrum of $L$ and the spectrum of $(A+D F(\bar{x}))_{0}$, which has been proved in Magal and Ruan [36, Proposition 4.22].

Proposition 3.4. Let the assumptions of Theorem 3.3 be satisfied. Let $\eta>0$ such that

$$
\sup _{\lambda \in \sigma_{s}\left(A_{0}\right)} \operatorname{Re}(\lambda)<-\eta<\eta<\inf _{\lambda \in \sigma_{u}\left(A_{0}\right)} \operatorname{Re}(\lambda) .
$$

Then, there exists $r_{1} \in\left(0, r^{*}\right)$ such that

$$
B_{X_{0}}\left(0, r_{1}\right) \subset \Omega .
$$

Assume that $\bar{x} \in B_{X_{0}}\left(0, r_{1}\right)$ is an equilibrium of $\{U(t)\}_{t \geq 0}$ (i.e. $\bar{x} \in D(A)$ and $\left.A \bar{x}+F(\bar{x})=0\right)$. Then

$$
\Pi_{0 h} \bar{x}=\Psi\left(\Pi_{0 c} \bar{x}\right)
$$

and $\Pi_{0 c} \bar{x}$ is an equilibrium of the reduced equation

$$
\frac{d x_{c}(t)}{d t}=A_{0 c} x_{c}(t)+\Pi_{c} F\left(x_{c}(t)+\Psi\left(x_{c}(t)\right)\right) .
$$


Moreover, consider the linearized equation at $\Pi_{0 c} \bar{x}$,

$$
\frac{d y_{c}(t)}{d t}=L(\bar{x}) y_{c}(t)
$$

with

$$
L(\bar{x})=\left[A_{0 c}+\Pi_{c} D F(\bar{x})\left[I+D \Psi\left(\Pi_{0 c} \bar{x}\right)\right]\right],
$$

we have the following spectral properties

$$
\sigma(L(\bar{x}))=\sigma\left((A+D F(\bar{x}))_{0}\right) \cap\{\lambda \in \mathbb{C}: \operatorname{Re}(\lambda) \in[-\eta, \eta]\} .
$$

\section{Proof of the Hopf bifurcation theorem}

To prove Theorem 2.4, we apply Theorem 3.3 and Proposition 3.4. In order to apply the reduction technics, we first incorporate the parameter into the state variable by considering the following system

$$
\left\{\begin{array}{l}
\frac{d \mu(t)}{d t}=0 \\
\frac{d u(t)}{d t}=A u(t)+F(\mu(t), u(t)) \\
(\mu(0), u(0))=\left(\mu_{0}, u_{0}\right) \in(-\varepsilon, \varepsilon) \times \overline{D(A)} .
\end{array}\right.
$$

Note that $F$ is only defined in a neighborhood of $(0,0) \in \mathbb{R} \times X$. In order to rewrite (4.1) as an abstract Cauchy problem, consider the Banach space $\mathbb{R} \times X$ endowed with the usual product norm

$$
\left\|\left(\begin{array}{l}
\mu \\
x
\end{array}\right)\right\|=\max (|\mu|,\|x\|)
$$

and the linear operator $\mathcal{A}: D(\mathcal{A}) \subset \mathbb{R} \times X \rightarrow \mathbb{R} \times X$ defined by

$$
\mathcal{A}\left(\begin{array}{l}
\mu \\
x
\end{array}\right)=\left(\begin{array}{c}
0 \\
A x+\partial_{\mu} F(0,0) \mu
\end{array}\right)=\left(\begin{array}{cc}
0 & 0 \\
\partial_{\mu} F(0,0) & A
\end{array}\right)\left(\begin{array}{l}
\mu \\
x
\end{array}\right)
$$

with

$$
D(\mathcal{A})=\mathbb{R} \times D(A) .
$$

Observe that by Assumption 2.3-(a) we have $\partial_{x} F(0,0)=0$, and the linear operator $\mathcal{A}$ is the generator of the linearized equation of system $(4.1)$ at $(0,0)$. Consider the function $\mathcal{F}:(-\varepsilon, \varepsilon) \times B_{X_{0}}(0, \varepsilon) \rightarrow \mathbb{R} \times X$ defined by

$$
\mathcal{F}\left(\begin{array}{l}
\mu \\
x
\end{array}\right)=\left(\begin{array}{c}
0 \\
F(\mu, x)-\partial_{\mu} F(0,0) \mu
\end{array}\right) .
$$

Using the variable $v(t)=\left(\begin{array}{l}\mu(t) \\ u(t)\end{array}\right)$, we can rewrite system (4.1) as the following abstract Cauchy problem

$$
\frac{d v(t)}{d t}=\mathcal{A} v(t)+\mathcal{F}(v(t)), t \geq 0, \quad v(0)=v_{0} \in \overline{D(\mathcal{A})}
$$

We first observe that $\mathcal{F}$ is defined on $B_{\mathbb{R} \times X}(0, \varepsilon)$ and is 4-time continuously differentiable. Moreover, using Assumption 2.3-(a), we have

$$
\mathcal{F}(0)=0 \quad \text { and } \quad D \mathcal{F}(0)=0 .
$$

In order to apply Theorem 3.3 and Proposition 3.4 to system (4.2), we need to verify Assumption 3.2. 


\subsection{State space decomposition for system (4.2)}

In order to apply the center manifold theorem, we need to study the spectral properties of the linear operator $\mathcal{A}$. From Assumption 2.3 (b) and (c), we know that

$$
\sigma\left(A_{0}\right) \cap i \mathbb{R}=\{\lambda(0), \overline{\lambda(0)}\} \text { and } \omega_{0, \text { ess }}\left(A_{0}\right)<0 .
$$

For each $\lambda_{0} \in \sigma\left(A_{0}\right)$ with $\operatorname{Re}\left(\lambda_{0}\right)>\omega_{0, \text { ess }}\left(A_{0}\right), \lambda_{0}$ is a pole of the resolvent of $A_{0}$. That is, there exists an integer $\widehat{k} \geq 1$ such that

$$
\left(\lambda I-A_{0}\right)^{-1}=\sum_{k=-\widehat{k}}^{\infty}\left(\lambda-\lambda_{0}\right)^{k} B_{k, \lambda_{0}}^{A_{0}}
$$

where

$$
B_{k, \lambda_{0}}^{A_{0}}:=\frac{1}{2 \pi i} \int_{S\left(\lambda_{0}, \varepsilon\right)^{+}}\left(\lambda-\lambda_{0}\right)^{-(k+1)}\left(\lambda I-A_{0}\right)^{-1} d \lambda
$$

for $\varepsilon>0$ small enough. The bounded linear operator $B_{-1, \lambda_{0}}^{A_{0}}$ is the projector on the generalized eigenspace of $A_{0}$ associated with $\lambda_{0}$.

Set

$$
\Pi_{0 c}^{A_{0}}=B_{-1, \lambda(0)}^{A_{0}}+B_{-1, \overline{\lambda(0)}}^{A_{0}}
$$

and

$$
\Pi_{0 u}^{A_{0}}=\sum_{\lambda \in \sigma\left(A_{0}\right): \operatorname{Re}(\lambda)>0} B_{-1, \lambda}^{A_{0}} .
$$

Since $\lambda(0)$ and $\overline{\lambda(0)}$ are simple eigenvalues of $A_{0}$, we have

$$
B_{-1, \gamma}^{A_{0}}=\lim _{\lambda \rightarrow \gamma}(\lambda-\gamma)\left(\lambda I-A_{0}\right)^{-1} \text { for } \gamma=\lambda(0) \text { or } \gamma=\overline{\lambda(0)} .
$$

Lemma 4.1. Let Assumptions 2.1 and 2.2 be satisfied. Then

$$
\sigma(\mathcal{A})=\sigma\left(\mathcal{A}_{0}\right)=\sigma\left(A_{0}\right) \cup\{0\}=\sigma(A) \cup\{0\},
$$

and for each $\lambda \in \rho(\mathcal{A})$,

$$
(\lambda I-\mathcal{A})^{-1}\left(\begin{array}{l}
\mu \\
x
\end{array}\right)=\left(\begin{array}{c}
\lambda^{-1} \mu \\
(\lambda I-A)^{-1}\left[x+\partial_{\mu} F(0,0) \lambda^{-1} \mu\right]
\end{array}\right) .
$$

Proof. Let $\lambda \in \mathbb{C} \backslash(\sigma(A) \cup\{0\})$. Then

$$
\begin{aligned}
& (\lambda I-\mathcal{A})\left(\begin{array}{c}
\mu \\
x
\end{array}\right)=\left(\begin{array}{c}
\widehat{\mu} \\
\widehat{x}
\end{array}\right) \\
& \Leftrightarrow\left(\begin{array}{c}
\lambda \mu \\
\lambda x-A x-\partial_{\mu} F(0,0) \mu
\end{array}\right)=\left(\begin{array}{c}
\widehat{\mu} \\
\widehat{x}
\end{array}\right) \\
& \Leftrightarrow\left\{\begin{array}{l}
\mu=\lambda^{-1} \widehat{\mu}, \\
x=(\lambda I-A)^{-1}\left[\widehat{x}+\partial_{\mu} F(0,0) \lambda^{-1} \widehat{\mu}\right] .
\end{array}\right.
\end{aligned}
$$

It follows that

$$
(\lambda I-\mathcal{A})^{-1}\left(\begin{array}{c}
\widehat{\mu} \\
\widehat{x}
\end{array}\right)=\left(\begin{array}{c}
\lambda^{-1} \widehat{\mu} \\
(\lambda I-A)^{-1}\left[\widehat{x}+\partial_{\mu} F(0,0) \lambda^{-1} \widehat{\mu}\right]
\end{array}\right),
$$

SO

$$
\rho(\mathcal{A}) \supset \mathbb{C} \backslash \sigma(A) \cup\{0\}
$$


It is clear that $0 \in \sigma(\mathcal{A})$ because

$$
\mathcal{A}\left(\begin{array}{c}
\mu \\
(-A)^{-1} \partial_{\mu} F(0,0) \mu
\end{array}\right)=\left(\begin{array}{l}
0 \\
0
\end{array}\right) .
$$

Moreover, if $\lambda \in \sigma(A)$, we have

$$
(\lambda I-\mathcal{A})\left(\begin{array}{c}
0 \\
x
\end{array}\right)=\left(\begin{array}{c}
0 \\
\widehat{x}
\end{array}\right) \Leftrightarrow(\lambda I-A) x=\widehat{x} .
$$

So $\lambda \in \sigma(\mathcal{A})$.

Lemma 4.2. Let Assumptions 2.1-2.2 be satisfied. The linear operator $\mathcal{A}: D(\mathcal{A}) \subset \mathbb{R} \times X \rightarrow \mathbb{R} \times X$ satisfies Assumptions 2.1-2.2. Moreover, we have

$$
T_{\mathcal{A}_{0}}(t)\left(\begin{array}{c}
\mu \\
x
\end{array}\right):=\left(\begin{array}{c}
\mu \\
T_{A_{0}}(t) x+S_{A}(t) \partial_{\mu} F(0,0) \mu
\end{array}\right)
$$

and

$$
S_{\mathcal{A}}(t)\left(\begin{array}{c}
\mu \\
x
\end{array}\right):=\left(\begin{array}{c}
t \mu \\
S_{A}(t) x+\int_{0}^{t} S_{A}(l) \partial_{\mu} F(0,0) \mu d l
\end{array}\right) .
$$

Furthermore,

$$
\omega_{0, \mathrm{ess}}\left(\mathcal{A}_{0}\right)=\omega_{0, \mathrm{ess}}\left(A_{0}\right) .
$$

Proof. To prove that $\mathcal{A}$ satisfies Assumptions 2.1-2.2 it is sufficient to apply Theorem 3.1 in Magal and Ruan [34]. Recall that

$$
\left(\lambda I-A_{0}\right)^{-1} x=\int_{0}^{+\infty} e^{-\lambda t} T_{A_{0}}(t) x d t
$$

and

$$
(\lambda I-A)^{-1} x=\lambda \int_{0}^{+\infty} e^{-\lambda t} S_{A}(t) x d t .
$$

Thus, for each $\lambda>0$ large enough,

$$
\int_{0}^{+\infty} e^{-\lambda t}\left(\begin{array}{c}
\mu \\
T_{A_{0}}(t) x+S_{A}(t) \partial_{\mu} F(0,0) \mu
\end{array}\right) d t=\left(\begin{array}{c}
\lambda^{-1} \mu \\
\left(\lambda I-A_{0}\right)^{-1} x+\lambda^{-1}(\lambda I-A)^{-1} \partial_{\mu} F(0,0) \mu
\end{array}\right)
$$

and

$$
\lambda \int_{0}^{+\infty} e^{-\lambda t}\left(\begin{array}{c}
t \mu \\
S_{A}(t) x+\int_{0}^{t} S_{A}(l) \partial_{\mu} F(0,0) \mu d l
\end{array}\right) d t=\left(\begin{array}{c}
\lambda^{-1} \mu \\
(\lambda I-A)^{-1} x+\lambda^{-1}(\lambda I-A)^{-1} \partial_{\mu} F(0,0) \mu
\end{array}\right) .
$$

It follows that $T_{\mathcal{A}_{0}}(t)$ and $S_{\mathcal{A}}(t)$ are defined, respectively, by (4.3) and (4.4).

Using formula (4.3), we deduce that

$$
\left\|T_{\mathcal{A}_{0}}(t)\right\|_{\mathrm{ess}}=\left\|T_{A_{0}}(t)\right\|_{\mathrm{ess}}, \quad \forall t \geq 0,
$$

(since $\mu \in \mathbb{R}$ ) and it follows that

$$
\omega_{0, \mathrm{ess}}\left(\mathcal{A}_{0}\right)=\lim _{t \rightarrow+\infty} \frac{\ln \left(\left\|T_{\mathcal{A}_{0}}(t)\right\|_{\mathrm{ess}}\right)}{t}=\lim _{t \rightarrow+\infty} \frac{\ln \left(\left\|T_{A_{0}}(t)\right\|_{\mathrm{ess}}\right)}{t}=\omega_{0, \mathrm{ess}}\left(A_{0}\right) .
$$

This completes the proof. 
Next, we compute the projectors on the generalized eigenspace associated with some eigenvalue of $\mathcal{A}$. Consider $\lambda_{0} \in\left\{\lambda \in \sigma(\mathcal{A}): \operatorname{Re}(\lambda)>\omega_{0, \text { ess }}\left(A_{0}\right)\right\} \backslash\{0\}$. Since

$$
\omega_{0, \mathrm{ess}}\left(\mathcal{A}_{0}\right)=\omega_{0, \mathrm{ess}}\left(A_{0}\right) \text { and } \sigma(\mathcal{A})=\sigma\left(\mathcal{A}_{0}\right)=\sigma\left(A_{0}\right) \cup\{0\}=\sigma(A) \cup\{0\},
$$

it follows that $\lambda_{0}$ is a pole of order $k_{0}$ of the resolvent of $\mathcal{A}_{0}$. Since $\operatorname{Re}\left(\lambda_{0}\right)>\omega_{0 \text {,ess }}\left(\mathcal{A}_{0}\right)$, by Lemma 3.11 in Magal and Ruan [36], we deduce that $\lambda_{0}$ is a pole of order $k_{0}$ of the resolvent of $\mathcal{A}$. Moreover, $\lambda_{0}$ is a pole of order $k_{1}$ of the resolvent of $A$. We have

$$
(\lambda I-\mathcal{A})^{-1}=\sum_{k=-k_{0}}^{\infty}\left(\lambda-\lambda_{0}\right)^{k} B_{k, \lambda_{0}}^{\mathcal{A}}
$$

and

$$
(\lambda I-A)^{-1}=\sum_{k=-k_{1}}^{\infty}\left(\lambda-\lambda_{0}\right)^{k} B_{k, \lambda_{0}}^{A}
$$

for $\left|\lambda-\lambda_{0}\right|$ small enough. The projector on the generalized eigenspace of $A$ (respectively, $\mathcal{A}$ ) associated with $\lambda_{0}$ is $B_{-1, \lambda_{0}}^{A}$ (respectively, $B_{-1, \lambda_{0}}^{\mathcal{A}}$ ).

We have $k_{1}=k_{0}$. Indeed, we have

$$
(\lambda I-\mathcal{A})^{-1}\left(\begin{array}{c}
\mu \\
x
\end{array}\right)=\left(\begin{array}{c}
\lambda^{-1} \mu \\
(\lambda I-A)^{-1}\left[x+\partial_{\mu} F(0,0) \lambda^{-1} \mu\right]
\end{array}\right)
$$

so

$$
\begin{aligned}
& \lim _{\lambda\left(\neq \lambda_{0}\right) \rightarrow \lambda_{0}}\left(\lambda-\lambda_{0}\right)^{k_{1}}(\lambda I-\mathcal{A})^{-1}\left(\begin{array}{c}
\mu \\
x
\end{array}\right) \\
& =\lim _{\lambda\left(\neq \lambda_{0}\right) \rightarrow \lambda_{0}}\left(\begin{array}{c}
\left(\lambda-\lambda_{0}\right)^{k_{1}} \lambda^{-1} \mu \\
\left(\lambda-\lambda_{0}\right)^{k_{1}}(\lambda I-A)^{-1}\left[x+\partial_{\mu} F(0,0) \lambda^{-1} \mu\right]
\end{array}\right) \\
& =\left(\begin{array}{c}
0 \\
B_{-k_{1}, \lambda_{0}}^{A}\left[x+\partial_{\mu} F(0,0) \lambda_{0}^{-1} \mu\right]
\end{array}\right) .
\end{aligned}
$$

Since the above limit exists, it follows that $k_{0} \leq k_{1}$, and since $B_{-k_{1}, \lambda_{0}}^{A} \neq 0$ it follows that $k_{0}=k_{1}$. So we obtain the following lemma.

Lemma 4.3. Let $\lambda_{0} \in\left\{\lambda \in \sigma(\mathcal{A}): \operatorname{Re}(\lambda)>\omega_{0 \text {,ess }}\left(A_{0}\right)\right\} \backslash\{0\}$. Then, $\lambda_{0}$ is a pole of order $k_{0}$ of the resolvent of $A$ if and only if $\lambda_{0}$ is a pole of order $k_{0}$ of the resolvent of $\mathcal{A}$.

Now we compute

$$
B_{k, \lambda_{0}}^{\mathcal{A}}:=\frac{1}{2 \pi i} \int_{S\left(\lambda_{0}, \varepsilon\right)^{+}}\left(\lambda-\lambda_{0}\right)^{-(k+1)}(\lambda I-\mathcal{A})^{-1} d \lambda .
$$

Set

$$
\left(\begin{array}{c}
\widehat{\mu} \\
\widehat{x}
\end{array}\right):=B_{k, \lambda_{0}}^{\mathcal{A}}\left(\begin{array}{l}
\mu \\
x
\end{array}\right)
$$


Since

$$
\begin{aligned}
(\lambda I-\mathcal{A})^{-1}\left(\begin{array}{c}
\mu \\
x
\end{array}\right)= & \left(\begin{array}{c}
\lambda^{-1} \mu \\
(\lambda I-A)^{-1}\left[x+\partial_{\mu} F(0,0) \lambda^{-1} \mu\right]
\end{array}\right) \\
= & \left(\sum_{k=-k_{0}}\left(\lambda-\lambda_{0}\right)^{k} B_{k, \lambda_{0}}^{A} x\right) \\
& +\left(\sum_{k=-k_{0}}\left(\lambda-\lambda_{0}\right)^{k} B_{k, \lambda_{0}}^{\lambda^{-1} \mu} \partial_{\mu} F(0,0) \lambda^{-1} \mu\right)
\end{aligned}
$$

it follows that

$$
\begin{gathered}
\widehat{\mu}=\frac{1}{2 \pi i} \int_{S_{\mathbb{C}}\left(\lambda_{0}, \varepsilon\right)^{+}}\left(\lambda-\lambda_{0}\right)^{-k-1} \lambda^{-1} \mu d \lambda \\
\widehat{x}=\sum_{j=-k_{0}} \frac{1}{2 \pi i} \int_{S_{\mathbb{C}}\left(\lambda_{0}, \varepsilon\right)^{+}}\left(\lambda-\lambda_{0}\right)^{-k-1}\left(\lambda-\lambda_{0}\right)^{j} B_{j, \lambda_{0}}^{A} x d \lambda \\
+\sum_{j=-k_{0}} \frac{1}{2 \pi i} \int_{S_{\mathbb{C}}\left(\lambda_{0}, \varepsilon\right)^{+}}\left(\lambda-\lambda_{0}\right)^{-k-1}\left(\lambda-\lambda_{0}\right)^{j} \lambda^{-1} B_{j, \lambda_{0}}^{A} \partial_{\mu} F(0,0) \mu d \lambda
\end{gathered}
$$

and

$$
\lambda^{-1}=\sum_{l=0}^{+\infty}\left(\lambda-\lambda_{0}\right)^{l} \frac{(-1)^{l}}{\lambda_{0}^{l+1}} .
$$

Since

$$
\begin{aligned}
& \frac{1}{2 \pi i} \int_{S_{\mathbb{C}}\left(\lambda_{0}, \varepsilon\right)^{+}}\left(\lambda-\lambda_{0}\right)^{-k-1}\left(\lambda-\lambda_{0}\right)^{j} \lambda^{-1} d \lambda \\
& \quad=\frac{1}{2 \pi i} \sum_{l=0}^{+\infty} \frac{(-1)^{l}}{\lambda_{0}^{l+1}} \int_{S_{\mathbb{C}}\left(\lambda_{0}, \varepsilon\right)^{+}}\left(\lambda-\lambda_{0}\right)^{[j+l-(k+1)]} d \lambda
\end{aligned}
$$

and

$$
\begin{aligned}
\int_{S_{\mathbb{C}}\left(\lambda_{0}, \varepsilon\right)^{+}}\left(\lambda-\lambda_{0}\right)^{[j+l-(k+1)]} d \lambda & =\int_{0}^{2 \pi}\left(\rho e^{i \theta}\right)^{[j+l-(k+1)]} i \rho e^{i \theta} d \theta \\
& =i \rho^{[j+l-k]} \int_{0}^{2 \pi}\left(e^{i \theta}\right)^{[j+l-k]} d \theta \\
& = \begin{cases}2 \pi i, & \text { if } l=k-j \\
0, & \text { otherwise, }\end{cases}
\end{aligned}
$$

it implies that

$$
\frac{1}{2 \pi i} \int_{S_{\mathbb{C}}\left(\lambda_{0}, \varepsilon\right)^{+}}\left(\lambda-\lambda_{0}\right)^{-k-1}\left(\lambda-\lambda_{0}\right)^{j} \lambda^{-1} d \lambda=\frac{(-1)^{k-j}}{\lambda_{0}^{k-j+1}} .
$$


For $l=0$, it yields

$$
\int_{S_{\mathbb{C}}\left(\lambda_{0}, \varepsilon\right)^{+}}\left(\lambda-\lambda_{0}\right)^{[j-(k+1)]} d \lambda= \begin{cases}2 \pi i, & \text { if } j=k \\ 0, & \text { otherwise }\end{cases}
$$

and

$$
\sum_{j=-k_{0}} \frac{1}{2 \pi i} \int_{S_{\mathbb{C}}\left(\lambda_{0}, \varepsilon\right)^{+}}\left(\lambda-\lambda_{0}\right)^{-k-1}\left(\lambda-\lambda_{0}\right)^{j} B_{j, \lambda_{0}}^{A} x d \lambda=B_{k, \lambda_{0}}^{A} x .
$$

Therefore, we obtain

$$
\widehat{\mu}= \begin{cases}\frac{(-1)^{k} \mu}{\lambda_{0}^{k+1}}, & k \geq 0 \\ 0, & k<0\end{cases}
$$

and

$$
\widehat{x}=B_{k, \lambda_{0}}^{A} x+\sum_{j=-k_{0}}^{k} \frac{(-1)^{k-j}}{\lambda_{0}^{k-j+1}} B_{j, \lambda_{0}}^{A} \partial_{\mu} F(0,0) \mu .
$$

From the above computation, we obtain the following lemma.

Lemma 4.4. We have the following:

(i) The projector on the generalized eigenspace of $\mathcal{A}$ associated with

$$
\lambda_{0} \in\{\lambda \in \sigma(\mathcal{A}): \operatorname{Re}(\lambda)>0\}
$$

a pole of order $k_{0}$ of the resolvent of $\mathcal{A}$ is given by

$$
B_{-1, \lambda_{0}}^{\mathcal{A}}\left(\begin{array}{c}
\mu \\
x
\end{array}\right)=\left(B_{-1, \lambda_{0}}^{A} x+\sum_{j=-k_{0}}^{-1} \frac{0}{\lambda_{0}^{-j}} B_{j, \lambda_{0}}^{A} \partial_{\mu} F(0,0) \mu\right) .
$$

(ii) $\lambda(0)$ and $\overline{\lambda(0)}$ are simple eigenvalues of $\mathcal{A}$ and the projectors on the generalized eigenspace of $\mathcal{A}$ associated with $\lambda(0)$ and $\overline{\lambda(0)}$ are given by

$$
B_{-1, \gamma}^{\mathcal{A}}\left(\begin{array}{l}
\mu \\
x
\end{array}\right)=\left(\begin{array}{c}
0 \\
B_{-1, \gamma}^{A}\left[x+\gamma^{-1} \partial_{\mu} F(0,0) \mu\right]
\end{array}\right) \text { for } \gamma=\lambda(0) \text { or } \gamma=\overline{\lambda(0)} .
$$

The projector on the generalized eigenspace of $\mathcal{A}$ associated with 0 is given in the following lemma.

Lemma 4.5. 0 is a simple eigenvalue of $\mathcal{A}$ and the projector on the generalized eigenspace of $\mathcal{A}$ associated with 0 is given by

$$
B_{-1,0}^{\mathcal{A}}\left(\begin{array}{l}
\mu \\
x
\end{array}\right)=\left(\begin{array}{c}
\mu \\
(-A)^{-1} \partial_{\mu} F(0,0) \mu
\end{array}\right)
$$

Proof. Since $0 \in \rho(A)$, it follows that

$$
\begin{aligned}
\lim _{\lambda \rightarrow 0} \lambda(\lambda I-\mathcal{A})^{-1}\left(\begin{array}{c}
\mu \\
x
\end{array}\right) & =\lim _{\lambda \rightarrow 0}\left(\begin{array}{c}
\mu \\
\lambda(\lambda I-A)^{-1} x+(\lambda I-A)^{-1} \partial_{\mu} F(0,0) \mu
\end{array}\right) \\
& =\left(\begin{array}{c}
\mu \\
(-A)^{-1} \partial_{\mu} F(0,0) \mu
\end{array}\right)=: \Pi_{0}\left(\begin{array}{c}
\mu \\
x
\end{array}\right) .
\end{aligned}
$$

This completes the proof. 
From the above results, we obtain a state space decomposition with respect to the spectral properties of the linear operator $\mathcal{A}$. More precisely, the projector on the unstable linear manifold is given by

$$
\Pi_{u}^{\mathcal{A}}=\sum_{\lambda \in \sigma(A): \operatorname{Re}(\lambda)>0} B_{-1, \lambda}^{\mathcal{A}}
$$

and the projector on the linear center manifold is defined by

$$
\Pi_{c}^{\mathcal{A}}=B_{-1,0}^{\mathcal{A}}+B_{-1, \lambda(0)}^{\mathcal{A}}+B_{-1, \overline{\lambda(0)}}^{\mathcal{A}} .
$$

Set

$$
\Pi_{s}^{\mathcal{A}}:=I-\left(\Pi_{c}^{\mathcal{A}}+\Pi_{u}^{\mathcal{A}}\right)
$$

\subsection{Proof of Theorem 2.4}

Using the results of Sect. 4.1, we deduce that $\mathcal{A}$ satisfies Assumption 3.2 and we can apply Theorem 3.3 to the system

$$
\frac{d v(t)}{d t}=\mathcal{A} v(t)+\mathcal{F}(v(t)), t \geq 0, \quad v(0)=v_{0} \in \overline{D(\mathcal{A})}
$$

Set

$$
\mathcal{X}_{0 c}=\Pi_{c}^{\mathcal{A}}(\mathbb{R} \times \overline{D(A)})
$$

and

$$
\mathcal{X}_{0 h}=\left(I-\Pi_{c}^{\mathcal{A}}\right)(\mathbb{R} \times \overline{D(A)}) .
$$

Using Theorem 3.3, we can find $\Psi \in C_{b}^{k}\left(\mathcal{X}_{0 c}, \mathcal{X}_{0 h}\right)$ such that the manifold

$$
M=\left\{x_{c}+\Psi\left(x_{c}\right): x_{c} \in \mathcal{X}_{0 c}\right\}
$$

is locally invariant by the semiflow generated by (4.5).

By applying $\Pi_{c}^{\mathcal{A}}$ to both sides of (4.5), we obtain the reduced system in $\mathcal{X}_{0 c}=\Pi_{c}^{\mathcal{A}}(\mathbb{R} \times X)$ :

$$
\frac{d}{d t}\left(\begin{array}{c}
\mu(t) \\
x_{c}(t)
\end{array}\right)=\mathcal{A}_{0 c}\left(\begin{array}{c}
\mu(t) \\
x_{c}(t)
\end{array}\right)+\Pi_{c}^{\mathcal{A}} \mathcal{F}\left(\left(\begin{array}{c}
\mu(t) \\
x_{c}(t)
\end{array}\right)+\Psi\left(\begin{array}{c}
\mu(t) \\
x_{c}(t)
\end{array}\right)\right)
$$

where

$$
\left(\begin{array}{c}
\mu(t) \\
x_{c}(t)
\end{array}\right)=\Pi_{c}^{\mathcal{A}}\left(\begin{array}{c}
\mu(t) \\
u(t)
\end{array}\right)
$$

Now, since $\left(\begin{array}{c}\mu \\ 0\end{array}\right)$ is a branch of the equilibrium of (4.5), it corresponds to a branch of the equilibrium $\left(\begin{array}{c}\mu \\ \bar{x}_{c}(\mu)\end{array}\right)=\Pi_{c}^{\mathcal{A}}\left(\begin{array}{c}\mu \\ 0\end{array}\right)$ of system (4.6). Applying Proposition 3.4, to system (4.5) and using Assumption 2.3 , we deduce that the spectrum of the linearized equation of $(4.6)$ around $\left(\begin{array}{c}\mu \\ \bar{x}_{c}(\mu)\end{array}\right)$ consists of

$$
\{0, \lambda(\mu), \bar{\lambda}(\mu)\}
$$

It follows that we can apply the Hopf bifurcation theorem in the book by Hassard et al. [21] to system (4.6). The proof of Theorem 2.4 is complete. 


\section{Applications}

In this section, we present some examples to demonstrate how our main results can be applied to study Hopf bifurcation in different types of equations. For the sake of simplicity, we only consider some applications in the Hille-Yosida case. Examples where the linear operator is not Hille-Yosida will be considered elsewhere. The first subsection is devoted to delay differential equations, and we will obtain a known result on Hopf bifurcation in this context. Then, we will turn to systems of age-structured models and will provide some new Hopf bifurcation results.

Here, the case of delay differential equations is chosen only as an illustration, while the application to age-structured models will provide some results which can be used in various contexts. We also refer to Chu et al. [10] for an application of Theorem 2.4 to parabolic equations.

\subsection{Delay differential equations}

For $r \geq 0$, let $C:=C\left([-r, 0] ; \mathbb{R}^{n}\right)$ be the Banach space of continuous functions from $[-r, 0]$ into $\mathbb{R}^{n}$ endowed with the supremum norm

$$
\|\varphi\|_{\infty}=\sup _{\theta \in[-r, 0]}\|\varphi(\theta)\|
$$

Consider the system of delay differential equation with parameter

$$
\left\{\begin{array}{l}
\frac{d x(t)}{d t}=B x(t)+f\left(\mu, x_{t}\right), \quad \forall t \geq 0, \\
x_{0}=\varphi \in C
\end{array}\right.
$$

where $\mu \in \mathbb{R}, x_{t} \in C$ satisfies $x_{t}(\theta)=x(t+\theta), B \in M_{n}(\mathbb{R})$ is an $n \times n$ real matrix, and $f: \mathbb{R} \times C \rightarrow \mathbb{R}^{n}$ is a $C^{k}$ map with $k \geq 4$.

As described in Liu et al. [32], by setting $v(t)=\left(\begin{array}{c}0 \\ x_{t}\end{array}\right)$, we can rewrite the delay differential Eq. (5.1) as the following abstract non-densely defined Cauchy problem on the Banach space $X=\mathbb{R}^{n} \times C$,

$$
\frac{d v(t)}{d t}=A v(t)+F(\mu, v(t)), \quad t \geq 0, \quad v(0)=\left(\begin{array}{c}
0_{\mathbb{R}^{n}} \\
\varphi
\end{array}\right) \in \overline{D(A)},
$$

where $A: D(A) \subset X \rightarrow X$ is the linear operator defined by

$$
A\left(\begin{array}{c}
0_{\mathbb{R}^{n}} \\
\varphi
\end{array}\right)=\left(\begin{array}{c}
-\varphi^{\prime}(0)+B \varphi(0) \\
\varphi^{\prime}
\end{array}\right)
$$

with

$$
D(A)=\left\{0_{\mathbb{R}^{n}}\right\} \times C^{1}\left([-r, 0], \mathbb{R}^{n}\right)
$$

and $F: \mathbb{R} \times \overline{D(A)} \rightarrow X$ is defined by

$$
F\left(\mu,\left(\begin{array}{c}
0_{\mathbb{R}^{n}} \\
\varphi
\end{array}\right)\right)=\left(\begin{array}{c}
f(\mu, \varphi) \\
0_{C}
\end{array}\right) .
$$

We assume that $f(\mu, 0)=0, \forall \mu \in \mathbb{R}$, and set

$$
L\left(\mu,\left(\begin{array}{c}
0_{\mathbb{R}^{n}} \\
\psi
\end{array}\right)\right)=\partial_{x} F(\mu, 0)\left(\begin{array}{c}
0_{\mathbb{R}^{n}} \\
\psi
\end{array}\right)=\left(\begin{array}{c}
\partial_{\varphi} f(\mu, 0) \psi \\
0_{C}
\end{array}\right)=:\left(\begin{array}{c}
\widehat{L}(\mu, \psi) \\
0_{C}
\end{array}\right) .
$$


By Proposition 3.11 in Liu et al. [32], we know that the linear operator $A+L(\mu,):. D(A) \rightarrow X$ is a Hille-Yosida operator. Moreover, $\omega_{0, \text { ess }}\left((A+L(\mu, .))_{0}\right)=-\infty$ and

$$
\begin{aligned}
\sigma(A+L(\mu, .)) & =\sigma\left((A+L(\mu, .))_{0}\right) \\
& =\sigma_{P}\left((A+L(\mu, .))_{0}\right) \\
& =\{\lambda \in \mathbb{C}: \operatorname{det}(\Delta(\mu, \lambda))=0\},
\end{aligned}
$$

where

$$
\Delta(\mu, \lambda):=\lambda I-B-\widehat{L}\left(\mu, e^{\lambda \cdot} I\right) .
$$

Hence, $A+L$ satisfies Assumptions 1.1, 1.2 and 1.3(c). In order to apply the Hopf bifurcation theorem obtained above to system (5.1), we need to make the following assumption.

Assumption 5.1. Let $\varepsilon>0$ and $f \in C^{k}\left((-\varepsilon, \varepsilon) \times B_{C}(0, \varepsilon) ; \mathbb{R}^{n}\right)$ for some $k \geq 4$. Assume that there exists a continuously differentiable map $\lambda:(-\varepsilon, \varepsilon) \rightarrow \mathbb{C}$ such that for each $\mu \in(-\varepsilon, \varepsilon)$,

$$
\operatorname{det}(\Delta(\mu, \lambda(\mu)))=0
$$

and $\lambda(\mu)$ is a simple eigenvalue of $\left(A+\partial_{x} F(\mu, 0)\right)_{0}$, which is equivalent to

$$
\lim _{\lambda \rightarrow \lambda(\mu)} \frac{\operatorname{det}(\Delta(\mu, \lambda))}{(\lambda-\lambda(\mu))} \neq 0
$$

and

$$
\operatorname{dim}(\mathcal{N}(\Delta(\mu, \lambda(\mu))))=1
$$

Moreover, assume that

$$
\operatorname{Im}(\lambda(0))>0, \quad \operatorname{Re}(\lambda(0))=0, \quad \frac{d \operatorname{Re}(\lambda(0))}{d \mu} \neq 0,
$$

and

$$
\{\lambda \in \Omega: \operatorname{det}(\Delta(\lambda, 0))=0\} \cap i \mathbb{R}=\{\lambda(0), \overline{\lambda(0)}\} .
$$

From Theorem 2.4 and the results in Liu et al. [32], we can derive the following Hopf bifurcation theorem for delay differential equations. We refer to the books of Hale [19], Hassard [21], Hale and Verduyn Lunel [20], Wu [50], Diekmann et al. [14], and Arino et al. [7] for more results on Hopf bifurcation in the context of delay differential equations. See also Adimy [1] and Adimy and Arino [2] for related results using integrated semigroup method.

Theorem 5.2. Let Assumption 5.1 be satisfied. Then, there exist a constant $\varepsilon^{*}>0$ and three $C^{k-1}$ maps, $\varepsilon \rightarrow \mu(\varepsilon)$ from $\left(0, \varepsilon^{*}\right)$ into $\mathbb{R}, \varepsilon \rightarrow \varphi_{\varepsilon}$ from $\left(0, \varepsilon^{*}\right)$ into $C$, and $\varepsilon \rightarrow \gamma(\varepsilon)$ from $\left(0, \varepsilon^{*}\right)$ into $\mathbb{R}$, such that for each $\varepsilon \in\left(0, \varepsilon^{*}\right)$ there exists a $\gamma(\varepsilon)$-periodic function $x_{\varepsilon} \in C^{k}\left(\mathbb{R}, \mathbb{R}^{n}\right)$, which is a solution of (5.1) for the parameter value $\mu=\mu(\varepsilon)$ and the initial value $\varphi=\varphi_{\varepsilon}$. Moreover, we have the following properties:

(i) There exist a neighborhood $N$ of 0 in $\mathbb{R}^{n}$ and an open interval $I$ in $\mathbb{R}$ containing 0 such that for $\widehat{\mu} \in I$ and any periodic solution $\widehat{x}(t)$ in $N$ with minimal period $\widehat{\gamma}$ close to $\frac{2 \pi}{\omega}$ of $(5.1)$ for the parameter value $\widehat{\mu}$, there exists $\varepsilon \in\left(0, \varepsilon^{*}\right)$ such that $\widehat{x}(t)=x_{\varepsilon}(t+\theta)$ (for some $\left.\stackrel{\omega}{\theta} \in[0, \gamma(\varepsilon))\right), \mu(\varepsilon)=\widehat{\mu}$, and $\gamma(\varepsilon)=\widehat{\gamma}$.

(ii) The map $\varepsilon \rightarrow \mu(\varepsilon)$ is a $C^{k-1}$ function and

$$
\mu(\varepsilon)=\sum_{n=1}^{\left[\frac{k-2}{2}\right]} \mu_{2 n} \varepsilon^{2 n}+O\left(\varepsilon^{k-1}\right), \quad \forall \varepsilon \in\left(0, \varepsilon^{*}\right),
$$

where $\left[\frac{k-2}{2}\right]$ is the integer part of $\frac{k-2}{2}$. 
(iii) The period $\gamma(\varepsilon)$ of $t \rightarrow u_{\varepsilon}(t)$ is a $C^{k-1}$ function and

$$
\gamma(\varepsilon)=\frac{2 \pi}{\omega}\left[1+\sum_{n=1}^{\left[\frac{k-2}{2}\right]} \gamma_{2 n} \varepsilon^{2 n}\right]+O\left(\varepsilon^{k-1}\right), \quad \forall \varepsilon \in\left(0, \varepsilon^{*}\right),
$$

where $\omega$ is the imaginary part of $\lambda(0)$ defined in Assumption 5.1.

Remark 5.3. In Assumption 5.1, if we only assume that $k \geq 2$ and replace condition (5.2) by

$$
\{\lambda \in \mathbb{C}: \operatorname{det}(\Delta(0, \lambda))=0\} \cap i \omega \mathbb{Z}=\{i \omega,-i \omega\}
$$

with $\omega=\operatorname{Im}(\lambda(0))$, then using Remark 2.5, we deduce that assertion (i) of Theorem 5.2 holds. So we derive a well-known Hopf bifurcation theorem for delay differential equations (see Hale [19, Theorem 1.1, p. 246], and Hale and Verduyn Lunel [20, Theorem 1.1, p. 332 ]).

\subsection{Age structured models}

The existence of non-trivial periodic solutions in age-structured models was studied in Prüss [40], Cushing [11], Swart [42], Kostova and Li [30], and Bertoni [8] and it was believed that such periodic solutions are induced by Hopf bifurcation. However, there is no general Hopf bifurcation theorem for age-structured models. In Magal and Ruan [36], we used the center manifold theorem to prove a Hopf bifurcation theorem for a specific age-structured model. In this subsection, our goal is to establish a general Hopf bifurcation theorem for a general class of age-structured models (Webb [49], Iannelli [24])

$$
\left\{\begin{array}{l}
\frac{\partial u}{\partial t}+\frac{\partial u}{\partial a}=-D(a) u(t, a)+M(\mu, u(t, .))(a), \quad a \geq 0, t \geq 0 \\
u(t, 0)=B(\mu, u(t, .)) \\
u(0, .)=u_{0} \in L^{1}\left((0,+\infty), \mathbb{R}^{n}\right)
\end{array}\right.
$$

where $\mu \in \mathbb{R}$ is a parameter, $D()=.\operatorname{diag}\left(d_{1}(),. \ldots, d_{n}().\right) \in L^{\infty}\left((0,+\infty), M_{n}\left(\mathbb{R}^{+}\right)\right), M: \mathbb{R} \times L^{1}((0,+\infty)$, $\left.\mathbb{R}^{n}\right) \rightarrow L^{1}\left((0,+\infty), \mathbb{R}^{n}\right)$ is the mortality function, and $B: \mathbb{R} \times L^{1}\left((0,+\infty), \mathbb{R}^{n}\right) \rightarrow \mathbb{R}^{n}$ is the birth function. Further assumptions on $M$ and $B$ will be given later.

We assume that there exists a smooth branch of equilibrium from which the bifurcation will occur.

Assumption 5.4. Assume that there exists a parameterized curve $\mu \rightarrow \bar{u}(\mu)($.$) from (-\varepsilon, \varepsilon)$ into $L^{1}\left((0,+\infty), \mathbb{R}^{n}\right)$ such that

$$
\begin{gathered}
\bar{u}(\mu) \in W^{1,1}\left((0,+\infty), \mathbb{R}^{n}\right), \\
\frac{\partial \bar{u}(\mu)(a)}{\partial a}=-D(a) \bar{u}(\mu)(a)+M(\mu, \bar{u}(\mu))(a) \text { for almost every } a \geq 0,
\end{gathered}
$$

and

$$
\bar{u}(\mu)(0)=B(\mu, \bar{u}(\mu)) .
$$

Here, we follow the results developed in Thieme [43] and Magal [33]. Consider the Banach space

$$
X=\mathbb{R}^{n} \times L^{1}\left((0,+\infty), \mathbb{R}^{n}\right),
$$

the linear operator $A: D(A) \subset X \rightarrow X$ defined by

$$
A\left(\begin{array}{l}
0 \\
\varphi
\end{array}\right)=\left(\begin{array}{c}
-\varphi(0) \\
-\varphi^{\prime}-D \varphi
\end{array}\right)
$$

with

$$
D(A)=\{0\} \times W^{1,1}\left((0,+\infty), \mathbb{R}^{n}\right)
$$


and the function $F: \mathbb{R} \times \overline{D(A)} \rightarrow X$ defined by

$$
F\left(\mu,\left(\begin{array}{l}
0 \\
\varphi
\end{array}\right)\right)=\left(\begin{array}{c}
B(\mu, \varphi) \\
M(\mu, \varphi)
\end{array}\right) .
$$

We observe that $A$ is non-densely defined since

$$
\overline{D(A)}=\{0\} \times L^{1}\left((0,+\infty), \mathbb{R}^{n}\right) \neq X .
$$

Setting $v(t)=\left(\begin{array}{c}0 \\ u(t, .)\end{array}\right)$, we can rewrite system (5.3) as the following non-densely defined abstract Cauchy problem

$$
\frac{d v(t)}{d t}=A v(t)+F(\mu, v(t)), t \geq 0, \quad v(0)=\left(\begin{array}{c}
0 \\
u_{0}
\end{array}\right) \in \overline{D(A)}
$$

We need to specify the type of nonlinearity we are considering here. So we make the following assumptions.

Assumption 5.5. Assume that there exists $\gamma \in L^{\infty}\left((0,+\infty), M_{p \times n}(\mathbb{R})\right)$ for integer $p \geq 1$ such that:

(a) (Birth function) The map $B: \mathbb{R} \times L^{1}\left((0,+\infty), \mathbb{R}^{n}\right) \rightarrow \mathbb{R}^{n}$ is 4-time continuously differentiable and has the following form

$$
B(\mu, \varphi)=\int_{0}^{+\infty} \beta\left(\mu, \int_{0}^{+\infty} \gamma(s) \varphi(s) d s\right)(a) \varphi(a) d a+\Theta\left(\mu, \int_{0}^{+\infty} \gamma(s) \varphi(s) d s\right),
$$

where $\Theta: \mathbb{R} \times \mathbb{R}^{p} \rightarrow \mathbb{R}^{n}$ and $\beta: \mathbb{R} \times \mathbb{R}^{p} \rightarrow L^{\infty}\left((0,+\infty), M_{n}(\mathbb{R})\right)$ are $C^{4}$ maps.

(b) (Mortality function) The map $M: \mathbb{R} \times L^{1}\left((0,+\infty), \mathbb{R}^{n}\right) \rightarrow L^{1}\left((0,+\infty), \mathbb{R}^{n}\right)$ is 4 -time continuously differentiable and has the following form

$$
M(\varphi)(a)=\widehat{M}\left(\mu, \int_{0}^{+\infty} \gamma(s) \varphi(s) d s\right)(a) \varphi(a)
$$

and

$$
\widehat{M}: \mathbb{R} \times \mathbb{R}^{p} \rightarrow L^{\infty}\left((0,+\infty), M_{n}(\mathbb{R})\right) .
$$

We observe that for $\lambda>\max _{i=1, \ldots, n} \sup _{a \geq 0} \operatorname{ess}\left(-d_{i}(a)\right), \lambda \in \rho(A)$, and (since the matrices $D(a)$ and $D(l)$ commutes)

$$
\begin{aligned}
& (\lambda I-A)^{-1}\left(\begin{array}{l}
\alpha \\
\psi
\end{array}\right)=\left(\begin{array}{l}
0 \\
\varphi
\end{array}\right) \\
& \Leftrightarrow \varphi(A)=e^{-\int_{0}^{a} \lambda+D(l) d l} \alpha+\int_{0}^{a} e^{-\int_{s}^{a} \lambda+D(l) d l} \psi(s) d s .
\end{aligned}
$$

The linear operator $A$ is a Hille-Yosida operator and $A_{0}$, the part of $A$ in $\overline{D(A)}$, is the infinitesimal generator of the strongly continuous semigroup $\left\{T_{A_{0}}(t)\right\}$ of bounded linear operators on $\overline{D(A)}$,

$$
T_{A_{0}}(t)\left(\begin{array}{c}
0 \\
\varphi
\end{array}\right)=\left(\begin{array}{c}
0 \\
\widehat{T}_{A_{0}}(t) \varphi
\end{array}\right)
$$

where

$$
\widehat{T}_{A_{0}}(t)(\varphi)(a)= \begin{cases}\exp \left(-\int_{a-t}^{a} D(l) d l\right) \varphi(a-t), & \text { if } a \geq t \\ 0, & \text { otherwise. }\end{cases}
$$


Notice that

$$
A\left(\begin{array}{c}
0 \\
\bar{u}(\mu)
\end{array}\right)+F\left(\mu,\left(\begin{array}{c}
0 \\
\bar{u}(\mu)
\end{array}\right)\right)=0 .
$$

So we make the following change of variables

$$
w(t):=v(t)-\left(\begin{array}{c}
0 \\
\bar{u}(\mu)
\end{array}\right)
$$

and obtain

$$
\frac{d w(t)}{d t}=A w(t)+G(\mu, w(t)), \quad t \geq 0, \quad w(0)=x \in \overline{D(A)}
$$

where

$$
G(\mu, x)=F\left(\mu, x+\left(\begin{array}{c}
0 \\
\bar{u}(\mu)
\end{array}\right)\right)-F\left(\mu,\left(\begin{array}{c}
0 \\
\bar{u}(\mu)
\end{array}\right)\right)
$$

Note that

$$
G(\mu, 0)=0, \quad \forall \mu \in(-\varepsilon, \varepsilon) .
$$

The linearized equation of (5.4) around the equilibrium $\left(\begin{array}{c}0 \\ \bar{u}(\mu)\end{array}\right)$ (or (5.5) around the equilibrium 0 ) is given by

$$
\frac{d \widehat{v}(t)}{d t}=A \widehat{v}(t)+\partial_{x} F\left(\mu,\left(\begin{array}{c}
0 \\
\bar{u}(\mu)
\end{array}\right)\right) \widehat{v}(t), \quad t \geq 0, \quad \widehat{v}(0)=x \in \overline{D(A)} .
$$

To simplify the notations, we set

$$
\bar{x}_{\mu}=\left(\begin{array}{c}
0 \\
\bar{u}(\mu)
\end{array}\right) .
$$

We first estimate the essential growth rate of the strongly continuous semigroup generated by $\left(A+\partial_{x} F\left(\mu, \bar{x}_{\mu}\right)\right)_{0}$, the part of $A+\partial_{x} F\left(\mu, \bar{x}_{\mu}\right): D(A) \subset X \rightarrow X$ in $\overline{D(A)}$. We observe that

$$
\partial_{x} F\left(\mu, \bar{x}_{\mu}\right)\left(\begin{array}{c}
0 \\
\varphi
\end{array}\right)=\left(\begin{array}{c}
\partial_{x} B(\mu, \bar{u}(\mu))(\varphi) \\
L_{1}(a) \varphi(a)+L_{2}\left(\int_{0}^{+\infty} \gamma(s) \varphi(s) d s\right)(a)
\end{array}\right),
$$

where $L_{1} \in L^{\infty}\left((0,+\infty), M_{n}(\mathbb{R})\right)$ is defined by

$$
L_{1}(a):=\widehat{M}\left(\mu, \int_{0}^{+\infty} \gamma(s) \bar{u}(\mu)(s) d s\right)(a)
$$

and

$$
L_{2}(\widehat{\gamma})(a)=\partial_{\widehat{\gamma}} H\left(\mu, \int_{0}^{+\infty} \gamma(s) \bar{u}(\mu)(s) d s\right)(a)(\widehat{\gamma}),
$$

here $H: \mathbb{R} \times \mathbb{R}^{p} \rightarrow L^{\infty}\left((0,+\infty), \mathbb{R}^{n}\right)$ is the map defined by

$$
H(\mu, \widehat{\gamma})(a)=\widehat{M}(\mu, \widehat{\gamma})(a) \bar{u}(\mu)(a) .
$$

We split $\partial_{x} F\left(\mu, \bar{x}_{\mu}\right)$ into the sum of two operators

$$
\partial_{x} F\left(\mu, \bar{x}_{\mu}\right)\left(\begin{array}{l}
0 \\
\varphi
\end{array}\right)=B\left(\begin{array}{l}
0 \\
\varphi
\end{array}\right)+C\left(\begin{array}{l}
0 \\
\varphi
\end{array}\right),
$$


where $B: \overline{D(A)} \subset X \rightarrow X$ is the bounded linear operator

$$
B\left(\begin{array}{l}
0 \\
\varphi
\end{array}\right)=\left(\begin{array}{c}
0 \\
L_{1}(.) \varphi(.)
\end{array}\right)
$$

and $C: \overline{D(A)} \subset X \rightarrow X$ is the compact bounded linear operator defined by

$$
C\left(\begin{array}{l}
0 \\
\varphi
\end{array}\right)=\left(\begin{array}{c}
\partial_{x} B(\mu, \bar{u}(\mu))(\varphi) \\
L_{2}\left(\int_{0}^{+\infty} \gamma(s) \varphi(s) d s\right)(a)
\end{array}\right)
$$

We consider the linear non-autonomous semiflow $\{U(a, s)\}_{a \geq s \geq 0} \subset M_{n}(\mathbb{R})$ on $\mathbb{R}^{n}$ generated by

$$
\begin{aligned}
\frac{d U(a, s) x}{d a} & =\left(-D(a)+L_{1}(a)\right) U(a, s) x \quad \text { for almost every } a \geq s \geq 0, \\
U(s, s) x & =x \in \mathbb{R}^{n} .
\end{aligned}
$$

This is, $a \rightarrow U(a, s) x$ is the unique solution from $(s,+\infty)$ into $\mathbb{R}^{n}$ of the integral equation

$$
U(a, s) x=e^{-\int_{s}^{a} D(r) d r} x+\int_{s}^{a} e^{-\int_{l}^{a} D(r) d r} L_{1}(l) U(l, s) x d l .
$$

We make an assumption on the estimation of $U(a, s)$.

Assumption 5.6. Assume that there exist two constants, $\nu>0$ and $M \geq 1$, such that

$$
\|U(a, s)\| \leq M e^{-\nu(a-s)}, \quad \forall a \geq s \geq 0 .
$$

Set

$$
\Omega:=\{\lambda \in \mathbb{C}: \operatorname{Re}(\lambda)>-\nu\} .
$$

Here, we use the same approach as in Thieme [45], Magal [33], or Magal and Ruan [34]. More precisely, for each $\lambda \in \Omega$ set

$$
\begin{aligned}
R_{\lambda}\left(\begin{array}{l}
\alpha \\
\psi
\end{array}\right) & =\left(\begin{array}{l}
0 \\
\varphi
\end{array}\right) \\
\Leftrightarrow \varphi(a) & =e^{-\lambda a} U(a, 0) \alpha+\int_{0}^{a} e^{-\lambda(a-s)} U(a, s) \psi(s) d s .
\end{aligned}
$$

Then, $R_{\lambda}$ is a pseudo-resolvent and

$$
R_{\lambda}=(\lambda I-(A+B))^{-1} .
$$

Moreover, for each $\omega_{A+B} \in(0, \nu)$, we can find $M_{A+B} \geq 1$ such that

$$
\left\|(\lambda I-(A+B))^{-1}\right\| \leq \frac{M_{A+B}}{\left(\lambda+\omega_{A+B}\right)^{n}}, \quad \forall \lambda>-\omega_{A+B}, \quad \forall n \geq 1 .
$$

Then, $(A+B)_{0}$, the part of $A+B$ in $\overline{D(A)}$, generates a strongly continuous semigroup $\left\{T_{(A+B)_{0}}(t)\right\}_{t \geq 0}$ on $\overline{D(A)}$, which is defined by

$$
T_{(A+B)_{0}}(t)\left(\begin{array}{l}
0 \\
\varphi
\end{array}\right)=\left(\begin{array}{c}
0 \\
\widehat{T}_{(A+B)_{0}}(t) \varphi
\end{array}\right)
$$

where

$$
\widehat{T}_{(A+B)_{0}}(t)(\varphi)(a)= \begin{cases}U(a, a-t) \varphi(a-t), & \text { if } a \geq t \\ 0, & \text { otherwise }\end{cases}
$$


From (5.7) we obtain

$$
\left\|T_{(A+B)_{0}}(t)\right\| \leq M_{A+B} e^{-\omega_{A+B} t}, \quad \forall t \geq 0 .
$$

Thus, for each $\omega_{A+B} \in(0, \nu)$,

$$
\omega_{0, \text { ess }}\left((A+B)_{0}\right) \leq \omega_{0}\left((A+B)_{0}\right) \leq-\omega_{A+B} .
$$

Now, since $C$ is a compact bounded linear operator, we can apply the perturbation results in Thieme [46] or in Ducrot et al. [15] to deduce that

$$
\omega_{0, \text { ess }}\left((A+B+C)_{0}\right) \leq-\omega_{A+B}, \quad \forall \omega_{A+B} \in(0, \nu) .
$$

We obtain the following proposition.

Proposition 5.7. Let Assumptions 5.4-5.6 be satisfied. Then

$$
\omega_{0, \mathrm{ess}}\left(\left(A+\partial_{x} F\left(0, \bar{x}_{0}\right)\right)_{0}\right)<0,
$$

that is, the essential growth rate of the strongly continuous semigroup $\left\{T_{\left(A+\partial_{x} F\left(0, \bar{x}_{0}\right)\right)_{0}}(t)\right\}_{t \geq 0}$ is strictly negative.

In order to apply Theorem 2.4, it remains to precise the spectral properties of $\left(A+\partial_{x} F\left(\mu, \bar{x}_{\mu}\right)\right)_{0}$. Let $\lambda \in \Omega$. Since $(\lambda I-(A+B))$ is invertible, it follows that $\left(\lambda I-\left(A+\partial_{x} F\left(\mu, \bar{x}_{\mu}\right)\right)\right)=(\lambda I-(A+B+C))$ is invertible if and only if $I-C(\lambda I-(A+B))^{-1}$ is invertible. Moreover, when $I-C(\lambda I-(A+B))^{-1}$ is invertible we have

$$
(\lambda I-(A+B+C))^{-1}=(\lambda I-(A+B))^{-1}\left[I-C(\lambda I-(A+B))^{-1}\right]^{-1} .
$$

Here, in order to compute the resolvent and to derive a characteristic equation, we need more details. We have

$$
\begin{aligned}
\partial_{\varphi} B(\mu, \bar{u}(\mu))(\varphi)= & \int_{0}^{+\infty} \beta_{\mu, \bar{u}(\mu)}(a) \varphi(a) d a \\
& +\int_{0}^{+\infty} L_{3}\left(\mu, \int_{0}^{+\infty} \gamma(s) \varphi(s) d s\right)(a) d a \\
& +D \Theta\left(\mu, \int_{0}^{+\infty} \gamma(s) \bar{u}(\mu)(s) d s\right)\left(\int_{0}^{+\infty} \gamma(s) \varphi(s) d s\right),
\end{aligned}
$$

where

$$
\beta_{\mu, \bar{u}(\mu)}(a)=\beta\left(\mu, \int_{0}^{+\infty} \gamma(s) \bar{u}(\mu)(s) d s\right)(a)
$$

and $L_{3}: \mathbb{R} \times \mathbb{R}^{p} \rightarrow L^{\infty}\left((0,+\infty), M_{n}(\mathbb{R})\right)$ is given by

$$
L_{3}(\mu, \widehat{\gamma})(a)=\partial_{\widehat{\gamma}} \widehat{H}\left(\mu, \int_{0}^{+\infty} \gamma(s) \bar{u}(\mu)(s) d s\right)(a)(\widehat{\gamma}),
$$

in which $\widehat{H}: \mathbb{R} \times \mathbb{R}^{p} \rightarrow L^{\infty}\left((0,+\infty), \mathbb{R}^{n}\right)$ is the map defined by

$$
\widehat{H}(\mu, \widehat{\gamma})(a)=\beta(\mu, \widehat{\gamma})(a) \bar{u}(\mu)(a) .
$$


Consider the system

$$
\left(I-C(\lambda I-(A+B))^{-1}\right)\left(\begin{array}{l}
\alpha \\
\varphi
\end{array}\right)=\left(\begin{array}{l}
\widehat{\alpha} \\
\widehat{\varphi}
\end{array}\right)
$$

or

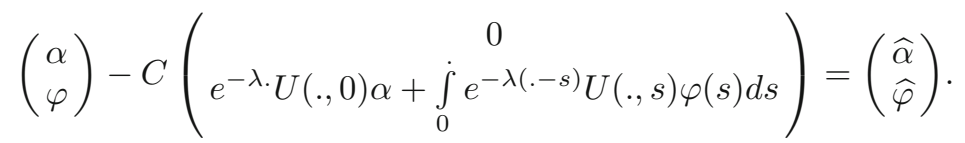

We obtain the system

$$
\left\{\begin{array}{l}
\alpha-\partial_{\varphi} B(\mu, \bar{u}(\mu))\left(e^{-\lambda \cdot} U(., 0) \alpha+\int_{0} e^{-\lambda(.-s)} U(., s) \varphi(s) d s\right)=\widehat{\alpha} \\
\varphi(a)-L_{2}\left(\int_{0}^{+\infty} \gamma(s)\left[e^{-\lambda s} U(s, 0) \alpha+\int_{0}^{s} e^{-\lambda(s-l)} U(s, l) \varphi(l) d l\right] d s\right)(a)=\widehat{\varphi}(a) .
\end{array}\right.
$$

Set

$$
\Gamma_{1}(\varphi)=\int_{0}^{+\infty} \beta_{\mu, \bar{u}(\mu)}(a) \varphi(a) d a
$$

and

$$
\Gamma_{2}(\varphi)=\int_{0}^{+\infty} \gamma(a) \varphi(a) d a
$$

We obtain

$$
\left.\left[I-\partial_{\varphi} B(\mu, \bar{u}(\mu)) e^{-\lambda \cdot} U(., 0) .\right)\right] \alpha-\partial_{\varphi} B(\mu, \bar{u}(\mu))\left(\int_{0} e^{-\lambda(.-s)} U(., s) \varphi(s) d s\right)=\widehat{\alpha} .
$$

By applying

$$
\varphi \rightarrow \Gamma_{1}\left(\int_{0}^{s} e^{-\lambda(s-l)} U(s, l) \varphi(l) d l\right)=: x_{1}
$$

and

$$
\varphi \rightarrow \Gamma_{2}\left(\int_{0}^{s} e^{-\lambda(s-l)} U(s, l) \varphi(l) d l\right)=: x_{2}
$$

to both sides of the second equation of system (5.9), we obtain

$$
\begin{aligned}
& {\left[I-\partial_{\varphi} B(\mu, \bar{u}(\mu))\left(e^{-\lambda \cdot} U(., 0) .\right)\right] \alpha} \\
& \quad-\left[x_{1}+\int_{0}^{+\infty} L_{3}\left(\mu, x_{2}\right)(a) d a+D \Theta\left(\mu, \int_{0}^{+\infty} \gamma(s) \bar{u}(\mu)(s) d s\right)\left(x_{2}\right)\right]
\end{aligned}
$$




$$
\begin{aligned}
= & \widehat{\alpha}, x_{1}-\Gamma_{1}\left(\int_{0} e^{-\lambda(.-l)} U(., l) L_{2}\left(x_{2}\right)(l) d l\right)-\Gamma_{1}\left(\int_{0} e^{-\lambda(.-l)} U(., l) L_{2}\left(\Gamma_{2}\left(e^{-\lambda \cdot} U(., 0) \alpha\right)\right)(l) d l\right) \\
= & \widehat{x}_{1}, x_{2}-\Gamma_{2}\left(\int_{0} e^{-\lambda(.-l)} U(., l) L_{2}\left(x_{2}\right)(l) d l\right) \\
& -\Gamma_{2}\left(\int_{0} e^{-\lambda(.-l)} U(., l) L_{2}\left(\Gamma_{2}\left(e^{-\lambda \cdot} U(., 0) \alpha\right)\right)(l) d l\right)=\widehat{x}_{2}
\end{aligned}
$$

where

$$
\widehat{x}_{1}:=\Gamma_{1}\left(\int_{0}^{s} e^{-\lambda(s-l)} U(s, l) \widehat{\varphi}(l) d l\right)
$$

and

$$
\widehat{x}_{2}:=\Gamma_{2}\left(\int_{0}^{s} e^{-\lambda(s-l)} U(s, l) \widehat{\varphi}(l) d l\right) .
$$

The above system can be rewritten as a finite dimensional system of linear equations

$$
\Delta(\mu, \lambda)\left(\begin{array}{c}
\alpha \\
x_{1} \\
x_{2}
\end{array}\right)=\left(\begin{array}{c}
\widehat{\alpha} \\
\widehat{x}_{1} \\
\widehat{x}_{2}
\end{array}\right)
$$

and we obtain the characteristic equation

$$
\operatorname{det}(\Delta(\mu, \lambda)):=0 .
$$

When $\Delta(\mu, \lambda)$ is invertible, we have

$$
\Delta(\mu, \lambda)^{-1}=\frac{1}{\operatorname{det}(\Delta(\mu, \lambda))}[\operatorname{cof}(\Delta(\mu, \lambda))]^{T},
$$

where $\operatorname{cof}(\Delta(\mu, \lambda))$ is the matrix of cofactors of $\Delta(\mu, \lambda)$. So we obtain

$$
\left(\begin{array}{c}
\alpha \\
x_{1} \\
x_{2}
\end{array}\right)=\frac{1}{\operatorname{det}(\Delta(\mu, \lambda))}[\operatorname{cof}(\Delta(\mu, \lambda))]^{T}\left(\begin{array}{c}
\widehat{\alpha} \\
\widehat{x}_{1} \\
\widehat{x}_{2}
\end{array}\right)
$$

We denote $[\operatorname{cof}(\Delta(\mu, \lambda))]^{T}$ by

$$
[\operatorname{cof}(\Delta(\mu, \lambda))]^{T}=\left(\begin{array}{lll}
N_{11} & N_{12} & N_{13} \\
N_{21} & N_{22} & N_{23} \\
N_{31} & N_{32} & N_{33}
\end{array}\right),
$$

where the blocks $N_{i j}$ are such that the system (5.11) can be rewritten as

$$
\left(\begin{array}{c}
\alpha \\
x_{1} \\
x_{2}
\end{array}\right)=\operatorname{det}(\Delta(\mu, \lambda))^{-1}\left(\begin{array}{l}
N_{11} \widehat{\alpha}+N_{12} \widehat{x}_{1}+N_{13} \widehat{x}_{2} \\
N_{21} \widehat{\alpha}+N_{22} \widehat{x}_{1}+N_{23} \widehat{x}_{2} \\
N_{31} \widehat{\alpha}+N_{32} \widehat{x}_{1}+N_{33} \widehat{x}_{2}
\end{array}\right) .
$$

Finally, using the second equation of (5.9), we have

$$
\varphi(a)=\widehat{\varphi}(a)+L_{2}\left(x_{2}\right)(a)+L_{2}\left(\int_{0}^{+\infty} \gamma(s) e^{-\lambda s} U(s, 0)(\alpha) d s\right)(a) .
$$


Therefore, we derive that if $\operatorname{det}(\Delta(\mu, \lambda)) \neq 0$, then $\left(I-C(\lambda I-(A+B))^{-1}\right)$ is invertible, and using Eqs. (5.9), (5.12), and (5.13), we obtain the following explicit formula

$$
\begin{aligned}
& \left(I-C(\lambda I-(A+B))^{-1}\right)\left(\begin{array}{c}
\alpha \\
\varphi
\end{array}\right)=\left(\begin{array}{c}
\widehat{\alpha} \\
\widehat{\varphi}
\end{array}\right) \\
& \Leftrightarrow\left\{\begin{array}{r}
\alpha=\operatorname{det}(\Delta(\mu, \lambda))^{-1}\left(N_{11} \widehat{\alpha}+N_{12} \widehat{x}_{1}+N_{13} \widehat{x}_{2}\right), \\
\varphi(a)=\widehat{\varphi}(a)+\operatorname{det}(\Delta(\mu, \lambda))^{-1} L_{2}\left(N_{31} \widehat{\alpha}+N_{32} \widehat{x}_{1}+N_{33} \widehat{x}_{2}\right)(a) \\
\quad+\operatorname{det}(\Delta(\mu, \lambda))^{-1} L_{2}\left(\int_{0}^{+\infty} \gamma(s) e^{-\lambda s} U(s, 0)\left(N_{11} \widehat{\alpha}+N_{12} \widehat{x}_{1}+N_{13} \widehat{x}_{2}\right) d s\right)(a) .
\end{array}\right.
\end{aligned}
$$

We observe that the only singularity in the above expression comes from $\operatorname{det}(\Delta(\mu, \lambda))^{-1}$ when $\lambda$ approaches an eigenvalue. By the above discussion, we obtain the following result.

Lemma 5.8. Let Assumptions 5.4-5.6 be satisfied. Then, we have the following:

(i) $\sigma(A+B+C) \cap \Omega=\sigma_{p}(A+B+C) \cap \Omega=\{\lambda \in \Omega: \operatorname{det}(\Delta(\mu, \lambda))=0\}$.

(ii) If $\lambda \in \rho(A+B+C)$, we have the following formula for the resolvent

$$
\begin{aligned}
(\lambda I & -(A+B+C))^{-1}\left(\begin{array}{c}
\widehat{\alpha} \\
\widehat{\varphi}
\end{array}\right)=\left(\begin{array}{c}
0 \\
\psi
\end{array}\right) \\
\Leftrightarrow & \psi(a)=e^{-\lambda a} U(a, 0) \operatorname{det}(\Delta(\mu, \lambda))^{-1}\left(N_{11} \widehat{\alpha}+N_{12} \widehat{x}_{1}+N_{13} \widehat{x}_{2}\right) \\
& \quad+\int_{0}^{a} e^{-\lambda(a-s)} U(a, s)\left[\widehat{\varphi}(a)+\operatorname{det}(\Delta(\mu, \lambda))^{-1} L_{2}\left(N_{31} \widehat{\alpha}+N_{32} \widehat{x}_{1}+N_{33} \widehat{x}_{2}\right)(a)\right. \\
& \left.+\operatorname{det}(\Delta(\mu, \lambda))^{-1} L_{2}\left(\int_{0}^{+\infty} \gamma(s) e^{-\lambda s} U(s, 0)\left(\begin{array}{c}
N_{11} \widehat{\alpha}+ \\
N_{12} \widehat{x}_{1}+N_{13} \widehat{x}_{2}
\end{array}\right) d s\right)(a)\right] d s
\end{aligned}
$$

where

$$
\begin{aligned}
& \widehat{x}_{1}=\int_{0}^{+\infty} \beta_{\mu, \bar{u}(\mu)}(a)\left(\int_{0}^{a} e^{-\lambda(a-l)} U(a, l) \widehat{\varphi}(l) d l\right) d a, \\
& \widehat{x}_{2}=\int_{0}^{+\infty} \gamma(a)\left(\int_{0}^{a} e^{-\lambda(a-l)} U(a, l) \widehat{\varphi}(l) d l\right) d a
\end{aligned}
$$

and $N_{i j}$ is defined in (5.11).

(iii) If $\lambda_{0} \in\{\lambda \in \Omega: \operatorname{det} \Delta(\mu, \lambda)=0\}=\Omega \cap \sigma(A+B+C)$, then $\lambda_{0}$ is isolated. Moreover, $\lambda_{0}$ is a simple pole of the resolvent of $A+B+C$ if

$$
\lim _{\lambda \rightarrow \lambda_{0}} \frac{\operatorname{det}(\Delta(\mu, \lambda))}{\lambda-\lambda_{0}} \neq 0 .
$$

Furthermore, $\lambda_{0}$ is a simple eigenvalue if in addition to condition (5.12) the dimension of the eigenspace of $A+B+C$ associated with $\lambda_{0}$ is 1 , which is equivalent to

$$
\operatorname{dim}\left(\mathcal{N}\left(\Delta\left(\lambda_{0}, \mu\right)\right)\right)=1 .
$$

Proof. Assume that $\lambda \in \Omega$ and $\operatorname{det}(\Delta(\lambda, \mu)) \neq 0$. From (5.6), (5.8), and (5.14) we obtain (5.15). Therefore, we obtain that $\{\lambda \in \Omega: \operatorname{det}(\Delta(\lambda, \mu)) \neq 0\} \subset \rho(A+B+C)$ and

$$
\sigma(A+B+C) \cap \Omega \subset\{\lambda \in \Omega: \operatorname{det}(\Delta(\lambda, \mu))=0\} .
$$


Conversely, assume that $\lambda \in \Omega$ and $\operatorname{det}(\Delta(\lambda, \mu))=0$. We claim that we can find $\left(\begin{array}{c}0 \\ \psi\end{array}\right) \in D(A) \backslash\{0\}$ such that

$$
(A+B+C)\left(\begin{array}{c}
0 \\
\psi
\end{array}\right)=\lambda\left(\begin{array}{c}
0 \\
\psi
\end{array}\right)
$$

Indeed, set

$$
\begin{aligned}
\left(\begin{array}{l}
\alpha \\
\varphi
\end{array}\right) & =(\lambda I-(A+B))\left(\begin{array}{l}
0 \\
\psi
\end{array}\right) \\
\Leftrightarrow\left(\begin{array}{l}
0 \\
\psi
\end{array}\right) & =(\lambda I-(A+B))^{-1}\left(\begin{array}{l}
\alpha \\
\varphi
\end{array}\right) .
\end{aligned}
$$

So we can find a solution of (5.16) if and only if we can find $\left(\begin{array}{c}\alpha \\ \varphi\end{array}\right) \in X \backslash\{0\}$ satisfying

$$
\left[I-C(\lambda I-(A+B))^{-1}\right]\left(\begin{array}{l}
\alpha \\
\varphi
\end{array}\right)=0 .
$$

Now from the above discussion, this is equivalent to find $\left(\alpha, x_{1}, x_{2}\right)^{T} \neq 0$ satisfying

$$
\Delta(\lambda, \mu)\left(\alpha, x_{1}, x_{2}\right)^{T}=0,
$$

where

$$
\begin{aligned}
& x_{1}=\int_{0}^{+\infty} \beta_{\mu, \bar{u}(\mu)}(a)\left(\int_{0}^{a} e^{-\lambda(a-l)} U(a, l) \varphi(l) d l\right) d a, \\
& x_{2}=\int_{0}^{+\infty} \gamma(a)\left(\int_{0}^{a} e^{-\lambda(a-l)} U(a, l) \varphi(l) d l\right) d a .
\end{aligned}
$$

But by the assumption $\operatorname{det}(\Delta(\lambda, \mu))=0$, we can find $\left(\begin{array}{c}0 \\ \psi\end{array}\right) \in D(A) \backslash\{0\}$ satisfying (5.16), which yields $\lambda \in \sigma_{p}(A+B+C)$. Hence, $\{\lambda \in \Omega: \operatorname{det}(\Delta(\lambda, \mu))=0\} \subset \sigma_{p}(A+B+C)$ and (i) follows. Assertion (iii) follows from (5.15) and the same argument as in the second of the above proof. The proof is complete.

From the above discussion we know that Assumptions 1.1, 1.2 and 1.3(c) hold. In order to apply the Hopf bifurcation theorem obtained above to system (5.3), we only need to make the following assumption.

Assumption 5.9. There exists a continuously differentiable map $\lambda:(-\varepsilon, \varepsilon) \rightarrow \mathbb{C}$ such that for each $\mu \in(-\varepsilon, \varepsilon)$,

$$
\operatorname{det}(\Delta(\mu, \lambda(\mu)))=0,
$$

and $\lambda(\mu)$ is a simple eigenvalue of $\left(A+\partial_{x} F\left(\mu, \bar{x}_{\mu}\right)\right)_{0}$ that is equivalent to verify that

$$
\lim _{\lambda \rightarrow \lambda(\mu)} \frac{\operatorname{det}(\Delta(\mu, \lambda))}{(\lambda-\lambda(\mu))} \neq 0
$$

and

$$
\operatorname{dim}(\mathcal{N}(\Delta(\mu, \lambda(\mu))))=1
$$

Moreover, assume that

$$
\operatorname{Im}(\lambda(0))>0, \quad \operatorname{Re}(\lambda(0))=0, \quad \frac{d \operatorname{Re}(\lambda(0))}{d \mu} \neq 0,
$$


and

$$
\{\lambda \in \Omega: \operatorname{det}(\Delta(\lambda, 0))=0\} \cap i \mathbb{R}=\{\lambda(0), \overline{\lambda(0)}\} .
$$

If $\lambda(\mu)$ is a solution of the characteristic equation, so is $\overline{\lambda(\mu)}$. So from the above assumption, we obtain a pair of conjugated simple eigenvalues. Now, using Theorem 2.4, we derive the following Hopf bifurcation theorem for system (5.3).

Theorem 5.10. Let the Assumptions 5.4-5.6 and 5.9 be satisfied. Then, there exist a constant $\varepsilon^{*}>0$ and three $C^{k-1}$ maps, $\varepsilon \rightarrow \mu(\varepsilon)$ from $\left(0, \varepsilon^{*}\right)$ into $\mathbb{R}, \varepsilon \rightarrow u_{0, \varepsilon}$ from $\left(0, \varepsilon^{*}\right)$ into $L^{1}\left((0,+\infty), \mathbb{R}^{n}\right)$, and $\varepsilon \rightarrow p(\varepsilon)$ from $\left(0, \varepsilon^{*}\right)$ into $\mathbb{R}$, such that for each $\varepsilon \in\left(0, \varepsilon^{*}\right)$ there exists a $p(\varepsilon)$-periodic function $u_{\varepsilon} \in$ $C^{k}\left(\mathbb{R}, L^{1}\left((0,+\infty), \mathbb{R}^{n}\right)\right)$, which is a solution of (5.3) for the parameter value $\mu=\mu(\varepsilon)$ and the initial value $u_{0}=u_{0, \varepsilon}$. Moreover, we have the following properties:

(i) There exist a neighborhood $N$ of 0 in $L^{1}\left((0,+\infty), \mathbb{R}^{n}\right)$ and an open interval $I$ in $\mathbb{R}$ containing 0 such that for $\widehat{\mu} \in I$ and any periodic solution $\widehat{u}(t)$ in $N$, with minimal period $\widehat{p}$ close to $\frac{2 \pi}{\operatorname{Im}(\lambda(0))}$, of $(5.3)$ for the parameter value $\widehat{\mu}$, there exists $\varepsilon \in\left(0, \varepsilon^{*}\right)$ such that $\widehat{u}(t)=u_{\varepsilon}(t+\theta)($ for some $\theta \in[0, p(\varepsilon)))$, $\mu(\varepsilon)=\widehat{\mu}$, and $p(\varepsilon)=\widehat{p}$.

(ii) The map $\varepsilon \rightarrow \mu(\varepsilon)$ is a $C^{k-1}$ function and

$$
\mu(\varepsilon)=\sum_{n=1}^{\left[\frac{k-2}{2}\right]} \mu_{2 n} \varepsilon^{2 n}+O\left(\varepsilon^{k-1}\right), \quad \forall \varepsilon \in\left(0, \varepsilon^{*}\right),
$$

where $\left[\frac{k-2}{2}\right]$ is the integer part of $\frac{k-2}{2}$.

(iii) The period $p(\varepsilon)$ of $t \rightarrow u_{\varepsilon}(t)$ is a $C^{k-1}$ function and

$$
p(\varepsilon)=\frac{2 \pi}{\operatorname{Im}(\lambda(0))}\left[1+\sum_{n=1}^{\left[\frac{k-2}{2}\right]} p_{2 n} \varepsilon^{2 n}\right]+O\left(\varepsilon^{k-1}\right), \quad \forall \varepsilon \in\left(0, \varepsilon^{*}\right),
$$

where $\operatorname{Im}(\lambda(0))$ is defined in Assumption 5.9.

Remark 5.11. If we only assume that $k \geq 2$, and the condition (5.17) is replaced by

$$
\{\lambda \in \mathbb{C}: \operatorname{det}(\Delta(0, \lambda))=0\} \cap i \omega \mathbb{Z}=\{i \omega,-i \omega\}
$$

with $\omega=\operatorname{Im}(\lambda(0))$, then using Remark 2.5, we deduce that assertion (i) of Theorem 5.10 holds.

\subsection{Coupled systems}

To conclude the paper, we would like to mention that the above theorem also applies to a coupled system of an ordinary differential equation and a partial differential equation of the form

$$
\left\{\begin{array}{l}
\frac{\partial u}{\partial t}+\frac{\partial u}{\partial a}=-D(a) u(t, a)+\widetilde{M}\left(\mu, \int_{0}^{+\infty} \widehat{\gamma}(a) u(t, a) d a, V(t)\right)(a) u(t, a) \\
u(t, 0)=B(\mu, u(t, .), V(t)) \\
\frac{d V(t)}{d t}=-C V(t)+G\left(\mu, V(t), \int_{0}^{+\infty} \widehat{\gamma}(a) u(t, a) d a\right) \\
u(0)=u_{0} \in L^{1}\left((0,+\infty), \mathbb{R}^{n}\right), \quad V(0)=V_{0} \in \mathbb{R}^{p}
\end{array}\right.
$$

where $B$ has the following form

$$
B(\mu, \varphi, V)=\int_{0}^{+\infty} \beta\left(\mu, \int_{0}^{+\infty} \widehat{\gamma}(s) \varphi(s) d s, V\right)(a) \varphi(a) d a+\Theta\left(\mu, \int_{0}^{+\infty} \widehat{\gamma}(s) \varphi(s) d s, V\right) .
$$


Indeed, in system (5.18), we can rewrite the ordinary differential equation as an age-structured model

$$
\left\{\begin{array}{l}
\frac{\partial v}{\partial t}+\frac{\partial v}{\partial a}=-C v(t, a) \\
v(t, 0)=G\left(\mu, \int_{0}^{+\infty} v(t, a) d a, \int_{0}^{+\infty} \widehat{\gamma}(a) u(t, a) d a\right) \\
v(0, .)=v_{0} \in L^{1}\left((0,+\infty), \mathbb{R}^{n}\right)
\end{array}\right.
$$

Then, by setting

$$
V(t):=\int_{0}^{+\infty} v(t, a) d a,
$$

we deduce that $V(t)$ satisfies the original ordinary differential equation

$$
\frac{d V(t)}{d t}=-C V(t)+G\left(\mu, V(t), \int_{0}^{+\infty} \widehat{\gamma}(a) u(t, a) d a\right) .
$$

Thus, by setting $w(t, a)=(u(t, a), v(t, a))$, we obtain a system of the form

$$
\left\{\begin{array}{l}
\frac{\partial w}{\partial t}+\frac{\partial w}{\partial a}=-\widehat{D}(a) w(t, a)+\widehat{M}\left(\mu, \int_{0}^{+\infty} \gamma(a) w(t, a) d a\right)(a) w(t, a) \\
w(t, 0)=\widehat{B}(\mu, w(t, .)) \\
w(0, .)=w_{0} \in L^{1}\left((0,+\infty), \mathbb{R}^{n} \times \mathbb{R}^{p}\right),
\end{array}\right.
$$

where

$$
\int_{0}^{+\infty} \gamma(a) w(t, a) d a=\left(\int_{0}^{+\infty} \widehat{\gamma}(a) u(t, a) d a, \int_{0}^{+\infty} v(t, a) d a\right) .
$$

So we can apply Theorem 5.10 to system (5.18). Following the above procedure, one can also apply Theorem 5.10 to study Hopf bifurcation in the coupled epidemic models for mutating pathogens considered by Li et al. [31].

\section{References}

1. Adimy, M.: Bifurcation de Hopf locale par semi-groupes intégrés. C. R. Acad. Sci. Paris Sér. I Math. 311, 423-428 (1990)

2. Adimy, M., Arino, O.: Bifurcation de Hopf globale pour des équations à retard par des semi-groupes intégrés. C. R. Acad. Sci. Paris Sér. I Math. 317, 767-772 (1993)

3. Andronov, A.A., Vitt, A.A., Khaikin, S.E.: Theory of Oscillators (in Russian), 2nd edn., Fizmatgiz, Moscow, 1959. English Edition, Pergamon Press, Oxford-New York-Toronto (1966)

4. Arendt, W.: Resolvent positive operators. Proc. Lond. Math. Soc. 54, 321-349 (1987)

5. Arendt, W.: Vector valued Laplace transforms and Cauchy problems. Israel J. Math. 59, 327-352 (1987)

6. Arendt, W., Batty, C.J.K., Hieber, M., Neubrander, F.: Vector-Valued Laplace Transforms and Cauchy Problems. Birkhäuser, Basel (2001)

7. Arino, O., Hbid, M.L., Ait Dads, E.: Delay Differential Equations and Applications. Springer, Berlin (2006)

8. Bertoni, S.: Periodic solutions for non-linear equations of structure populations. J. Math. Anal. Appl. 220, 250-267 (1998)

9. Crandall, M.G., Rabinowitz, P.H.: The Hopf bifurcation theorem in infinite dimensions. Arch. Ration. Mech. Anal. 67, 53-72 (1977)

10. Chu, J., Ducrot, A., Magal, P., Ruan, S.: Hopf bifurcation in a model with diffusion and non-linear boundary conditions for structured population dynamics. J. Differ. Equ. 247, 956-1000 (2009)

11. Cushing, J.M.: Bifurcation of time periodic solutions of the McKendrick equations with applications to population dynamics. Comput. Math. Appl. 9, 459-478 (1983)

12. Da Prato, G., Lunardi, A.: Stability, instability and center manifold theorem for fully nonlinear autonomous parabolic equations in Banach spaces. Arch. Ration. Mech. Anal. 101, 115-141 (1988) 
13. Da Prato, G., Sinestrari, E.: Differential operators with non-dense domain. Ann. Scuola. Norm. Sup. Pisa Cl. Sci 14, 285-344 (1987)

14. Diekmann, O., van Gils, S.A., Verduyn Lunel, S.M., Walther, H.-O.: Delay Equations: Functional-, Complex-, and Nonlinear Analysis. Springer, New York (1995)

15. Ducrot, A., Liu, Z., Magal, P.: Essential growth rate for bounded linear perturbation of non-densely defined Cauchy problems. J. Math. Anal. Appl 341, 501-518 (2008)

16. Friedrichs, K.O.: Advanced Ordinary Differential Equations. Gordon and Breach, New York (1965)

17. Golubitsky, M., Rabinowitz, P.H.: A sketch of the Hopf bifurcation theorem. In: Morawetz, C. S., Serrin, J. B., Sinai, Y. G. (eds.) Selected Works of Eberhard Hopf with Commentaries, pp. 111-118. American Mathematical Society, Providence (2002)

18. Hale, J.K.: Ordinary Differential Equations. Wiley, NewYork (1969)

19. Hale, J.K.: Theory of Function Differential Equations. Springer, New York (1977)

20. Hale, J.K., Verduyn Lunel, S.M.: Introduction to Functional Differential Equations. Springer, New York (1993)

21. Hassard, B.D., Kazarinoff, N.D., Wan, Y.-H.: Theory and Applications of Hopf Bifurcaton, London Mathematical Society Lecture Note Series, vol. 41. Cambridge University Press, Cambridge (1981)

22. Henry, D.: Geometric Theory of Semilinear Parabolic Equations, Lecture Notes in Mathematics. Springer, Berlin (1981)

23. Hopf, E.: Abzweigung einer periodischer Lösung von einer stationären Lösung eines Differentialsystems. Ber. Verh. Sächs. Akad. Wiss. Leipzig. Math-Nat. K1 95, 3-22 (1943)

24. Iannelli, M.: Mathematical Theory of Age-Structured Population Dynamics, Applied Mathematics Monographs, CNR, vol. 7. Giadini Editori e Stampatori, Pisa (1994)

25. Iooss, G.: Existence et stabilité de la solution périodique secondaire intervenant dans les problèmes d'évolution du type Navier-Stokes. Arch. Ration. Mech. Anal. 47, 301-329 (1972)

26. Iudovich, V.I.: The onset of auto-oscillations in a fluid. J. Appl. Math. Mech. 35, 587-603 (1971)

27. Joseph, D.D., Sattinger, D.H.: Bifurcating time periodic solutions and their stability. Arch. Ration. Mech. Anal. 45, 79-109 (1972)

28. Kellermann, H., Hieber, M.: Integrated semigroups. J. Funct. Anal. 84, 160-180 (1989)

29. Kielhőfer, H.: Bifurcation Theory: An Introduction with Applications to PDEs. Springer, New York (2004)

30. Kostova, T., Li, J.: Oscillations and stability due to juvenile competitive effects on adult fertility. Comput. Math. Appl. 32(11), 57-70 (1996)

31. Li, J., Zhou, Y., Ma, Z., Hyman, J.M.: Epidemiological models for mutating pathogens. SIAM J. Appl. Math. 65, $1-23(2004)$

32. Liu, Z., Magal, P., Ruan, S.: Projectors on the generalized eigenspaces for functional differential equations using integrated semigroups. J. Differ. Equ. 244, 1784-1809 (2008)

33. Magal, P.: Compact attractors for time-periodic age structured population models. Electron. J. Differ. Equ. 2001, $1-35(2001)$

34. Magal, P., Ruan, S.: On integrated semigroups and age structured models in $L^{p}$ spaces. Differ. Integral Equ. 20, 137-139 (2007)

35. Magal, P., Ruan, S.: On semilinear Cauchy problems with non-dense domain. Adv. Differ. Equ. 14(11/12), 1041-1084 (2009)

36. Magal, P., Ruan, S.: Center manifolds for semilinear equations with non-dense domain and applications on Hopf bifurcation in age structured models. Mem. Amer. Math. Soc. 202(951) (2009)

37. Marsden, J.: The Hopf bifurcation for nonlinear semigroups. Bull. Amer. Math. Soc. 79, 537-541 (1973)

38. Marsden, J., McCraken, M.: The Hopf Bifurcation and its Applications. Springer, New York (1976)

39. Neubrander, F.: Integrated semigroups and their application to the abstract Cauchy problem. Pac. J. Math. 135, 111-155 (1988)

40. Prüss, J.: On the qualitative behavior of populations with age-specific interactions. Comput. Math. Appl. 9, 327-339 (1983)

41. Sattinger, D.H.: Bifurcation of periodic solutions of the Navier-Stokes equations. Arch. Ration. Mech. Anal. 41, 66-80 (1971)

42. Swart, J.H.: Hopf bifurcation and the stability of non-linear age-dependent population models. Comput. Math. Appl. 15, 555-564 (1988)

43. Thieme, H.R.: Semiflows generated by Lipschitz perturbations of non-densely defined operators. Differ. Integral Equ. 3, 1035-1066 (1990)

44. Thieme, H.R.: "Integrated semigroups" and integrated solutions to abstract Cauchy problems. J. Math. Anal. Appl. 152, 416-447 (1990)

45. Thieme, H.R.: Analysis of age-structured population models with an additional structure. In: Arino, O., Axelrod, D.E., Kimmel, M. (eds.) Mathematical Population Dynamics, Lecture Notes in Pure and Applied Mathematics, vol. 131, pp. 115-126. Marcel Dekker, New York (1991) 
46. Thieme, H.R. : Quasi-compact semigroups via bounded perturbation. In: Arino, O., Axelrod, D., Kimmel, M. (eds.) Advances in Mathematical Population Dynamics-Molecules, Cells and Man, pp. 691-713. World Scientific, Singapore (1997)

47. Thieme, H.R.: Differentiability of convolutions, integrated semigroups of bounded semi-variation, and the inhomogeneous Cauchy problem. J. Evol. Equ. 8, 1-23 (2008)

48. Vanderbauwhede, A., Iooss, G. : Center manifold theory in infinite dimensions. In: Jones, C.K.R.T., Kirchgraber, U., Walther, H.O. (eds.) Dynamics Reported (new series), vol. 1, pp. 125-163. Springer, Berlin (1992)

49. Webb, G.F.: Theory of Nonlinear Age-Dependent Population Dynamics. Marcel Dekker, New York (1985)

50. Wu, J.: Theory and Applications of Partial Differential Equations. Springer, New York (1996)

Z. Liu

School of Mathematical Sciences

Beijing Normal University

Beijing 100875

People's Republic of China

P. Magal

UMR CNRS 5251, IMB \& INRIA sud-ouest Anubis

Université de Bordeaux

146 rue Léo Saignat

33076 Bordeaux

France

S. Ruan

Department of Mathematics

University of Miami,

Coral Gables

FL 33124-4250

USA

Email:ruan@math.miami.edu

(Received: 15 October 2009) 